\title{
Dynamic subfilter-scale stress model for large-eddy simulations
}

\author{
A. Rouhi and U. Piomelli* \\ Department of Mechanical and Materials Engineering, Queen's University, Kingston, \\ Ontario, Canada K7L $4 L 9$
}

B. J. Geurts

Department of Applied Mathematics, University of Twente, P.O. Box 217, 7500 AE Enschede, The Netherlands

(Received 13 January 2016; published 1 August 2016)

\begin{abstract}
We present a modification of the integral length-scale approximation (ILSA) model originally proposed by Piomelli et al. [Piomelli et al., J. Fluid Mech. 766, 499 (2015)] and apply it to plane channel flow and a backward-facing step. In the ILSA models the length scale is expressed in terms of the integral length scale of turbulence and is determined by the flow characteristics, decoupled from the simulation grid. In the original formulation the model coefficient was constant, determined by requiring a desired global contribution of the unresolved subfilter scales (SFSs) to the dissipation rate, known as SFS activity; its value was found by a set of coarse-grid calculations. Here we develop two modifications. We define a measure of SFS activity (based on turbulent stresses), which adds to the robustness of the model, particularly at high Reynolds numbers, and removes the need for the prior coarse-grid calculations: The model coefficient can be computed dynamically and adapt to large-scale unsteadiness. Furthermore, the desired level of SFS activity is now enforced locally (and not integrated over the entire volume, as in the original model), providing better control over model activity and also improving the near-wall behavior of the model. Application of the local ILSA to channel flow and a backward-facing step and comparison with the original ILSA and with the dynamic model of Germano et al. [Germano et al., Phys. Fluids A 3, 1760 (1991)] show better control over the model contribution in the local ILSA, while the positive properties of the original formulation (including its higher accuracy compared to the dynamic model on coarse grids) are maintained. The backward-facing step also highlights the advantage of the decoupling of the model length scale from the mesh.
\end{abstract}

DOI: 10.1103/PhysRevFluids.1.044401

\section{INTRODUCTION}

Large-eddy simulation (LES) is based on resolving the large energy-carrying eddies and parametrizing the unresolved ones. Filtering the governing equations of the fluid flow (conservation of mass, momentum, scalar, etc.) yields the equations governing the dynamics of the resolved scales [1]. The characteristic length of the filtering operation, the filter width $\Delta$ (also called turbulence resolution length scale by Pope [2]), defines the size of the smallest eddies retained in the calculation. The nonlinear interaction between different scales of turbulence does not allow the dynamics of the unresolved (subfilter) field to be fully separated from the resolved one. Consequently, the filtered momentum equation contains the contribution of unresolved eddies to the resolved field, which acts as an additional stress term. These stresses are commonly known as subgrid-scale stresses, but are called subfilter-scale (SFS) stresses here. The SFS stresses are usually modeled using an eddy-viscosity hypothesis and the model length scale is related to the filter width (in most commonly used models, the velocity scale also depends on $\Delta[3,4])$. Therefore, the filter width plays an important role in modeling the dynamics of the subfilter eddies, which in turn affect the resolved field.

\footnotetext{
*ugo@queensu.ca
} 
It is common practice to relate $\Delta$ to the grid size $h$. Pope [2] points out two problems related to this choice. First, when $\Delta \propto h$, the dynamics of the resolved field are linked to the numerical scheme, altering the LES from a physical model to a numerical procedure; furthermore, convergence to the solution of the LES equations is never reached. Second, the dependence of $\Delta$ on the grid topology makes LES an incomplete model. Vanella et al. [5], for instance, observed that discontinuities appear in the eddy viscosity when local refinement is used. These discontinuities may cause numerical instabilities and do not always reflect the flow physics; a refined treatment would require explicit accounting of commutator errors associated with strongly varying filter widths [6]. Piomelli et al. [7] discuss these issues in depth.

An alternative definition of $\Delta$ decouples the filter width from the grid [7]. In this paper, the authors propose a model length scale $\ell$ that is instead related to the turbulence itself, by calculating an approximation of the local integral length scale $L_{\text {est }}$ and setting $\Delta \propto L_{\text {est }}$ [integral length-scale approximation (ILSA)]. In this way, the LES is turned into a physical model for turbulence and full independent control is possible over numerical errors. Piomelli et al. [7] estimated $L_{\text {est }}$ based on the resolved turbulent kinetic energy and total dissipation; the single model constant $C_{k}$ was determined by requiring that the SFSs contribute a given amount to the total dissipation [8]. Decreasing $C_{k}$ is equivalent to decreasing the filter width and hence enhancing the physical resolution. More grid points are then required to resolve the smallest scale in the flow, as the number of grid cells covering the smallest resolved volume, which is proportional to $\Delta^{3}$, should be kept constant in this philosophy. Increasing the grid resolution, however, does not result in significant changes in the filter size once the local integral scale is reliably captured. In fact, a grid-converged solution can be achieved when $L_{\text {est }} / h$ is large [9]. Conversely, larger values of $C_{k}$ correspond to a wider range of modeled scales; grid convergence is achieved with coarser meshes, but the SFS model must give a more significant contribution to the transport and modeling errors may become more significant. Seen from this perspective, LES would be an act of balancing errors controlled fully by the user of the model [10].

In Ref. [7] $C_{k}$ is a global constant, calculated once, before the production simulation is performed. A target value for the chosen measure of SFS activity [8], $s_{\varepsilon}$ (the ratio of the SFS dissipation to the total, viscous and SFS, one), was assigned and the corresponding $C_{k}$ was obtained by performing several coarse-grid calculations, using the successive inverse polynomial interpolation approach for computational error reduction [11]. Successive inverse polynomial interpolation is based on the definition of a cost function $\phi$ measuring the difference between a target value of a given quantity (here the assigned $s_{\varepsilon}$ ) and the LES prediction of the same quantity. The method consists of three LES simulations on a coarse mesh for values of $C_{k}$ that are expected to bracket the optimum value [one at $C_{k}=0$ corresponding to the coarse direct numerical simulation (DNS), the second at a fairly large $C_{k}$, and the third with an intermediate value]. An interpolating parabola is constructed using the three predicted $\phi$ and the $C_{k}$ that yields the minimum $\phi$ is found. A LES using the optimized $C_{k}$ is carried out and the corresponding $\phi$ is predicted. If the $\phi$ thus obtained is not small enough (within a given tolerance), the interpolation is repeated using the newest $C_{k}$ and the nearest points. With this method the optimum $C_{k}$ is usually found with less than six simulations. The optimal model coefficient obtained in this way is subsequently used in production calculations. This formulation of the model, in which the overall integrated contribution of the SFS to the transport is used to set the constant, will be referred to as the global ILSA model. It was tested in channel flow and homogenous isotropic turbulence, with excellent agreement with the data; furthermore, several positive features of the model (such as the grid independence) were demonstrated.

Further key improvements are needed to make the ILSA model applicable to complex problems. First, for instance, as $\operatorname{Re} \rightarrow \infty$, the relative contribution of the SFS to the dissipation asymptotically approaches unity. Using $s_{\varepsilon}$ to determine $C_{k}$ would cause a loss of sensitivity to the SFS activity in this limit. Second, setting the integral of the SFS contribution to a single prespecified value may not be the best choice in flows with strong inhomogeneities: In those cases, the SFS contribution may be excessive in some regions (for instance, in thin shear layers), while in other regions the flow can be overresolved and correspondingly the SFS contribution would be low. Third, alternative methods of determining $C_{k}$ that do not require additional calculations would be beneficial; adjusting 
$C_{k}$ to the local state of the flow may be particularly useful in cases in which large-scale unsteadiness may change the state of the turbulence to first order (oscillating flows in the transitional state, for instance).

In this study we modify the ILSA model to make it more universally applicable to complex problems and address three key issues with the global ILSA formulation: (i) sensitivity at high $\mathrm{Re}$, (ii) suitability for nonhomogeneous flows, and (iii) computational efficiency. In particular, we adopt an alternative measure of SFS activity based on the contribution of the SFS directly to the turbulent stresses, instead of indirectly via dissipation. Furthermore, we enforce a desired level of SFS activity on a local basis. This provides better control over model activity, more suitable for flows that contain nonequilibrium effects. Finally, the model coefficient (which is not constant any longer) is calculated dynamically during the simulation, removing the need for preliminary simulations and better matching the SFS model to the local state of the flow. This model will be called the local ILSA.

In the following, the governing equations and the numerical method used are described. Then the local implementation of the ILSA model is introduced. The relationship between the chosen measure of SFS activity and more intuitive quantities (such as the SFS contribution to the turbulent kinetic energy or to the momentum transport) is discussed at some length. Then the proposed model is applied to plane channel flow at $\operatorname{Re}_{\tau}=1000$ and 2000 and flow over a backward-facing step (BFS). Final remarks and suggestions for future development will conclude the article.

\section{PROBLEM FORMULATION}

\section{A. Governing equations}

In this paper, the filtered equations of conservation of mass and momentum are solved:

$$
\begin{gathered}
\frac{\partial \bar{u}_{i}}{\partial x_{i}}=0 \\
\frac{\partial \bar{u}_{i}}{\partial t}+\frac{\partial\left(\bar{u}_{i} \bar{u}_{j}\right)}{\partial x_{j}}=-\frac{1}{\rho} \frac{\partial \bar{p}}{\partial x_{i}}+v \frac{\partial^{2} \bar{u}_{i}}{\partial x_{j} \partial x_{j}}-\frac{\partial \tau_{i j}}{\partial x_{j}}+f_{i},
\end{gathered}
$$

where $x_{1}, x_{2}$, and $x_{3}$ (also referred to as $x, y$, and $z$ ) are the streamwise, wall-normal, and spanwise directions, which correspond to the velocity components $\bar{u}_{1}, \bar{u}_{2}$, and $\bar{u}_{3}$ (or $\bar{u}, \bar{v}$, and $\bar{w}$ ), respectively. The unresolved SFS stresses $\tau_{i j}=\overline{u_{i} u_{j}}-\bar{u}_{i} \bar{u}_{j}$ constitute the central closure problem in LES. They are modeled using an eddy-viscosity approach

$$
\tau_{i j}^{a}=\tau_{i j}-\frac{\delta_{i j}}{3} \tau_{k k}=-2 v_{\mathrm{SFS}} \bar{S}_{i j}
$$

where $\bar{S}_{i j}=1 / 2\left(\partial \bar{u}_{i} / \partial x_{j}+\partial \bar{u}_{j} / \partial x_{i}\right)$ is the resolved strain-rate tensor and $\nu_{\mathrm{SFS}}$ is the eddy viscosity. Finally, $f_{i}$ is a body force. In forced homogeneous isotropic turbulence, the linear forcing $f_{i}=A \bar{u}_{i}$ was used to sustain turbulence following Rosales and Meneveau [12] (where $A$ is a proportionality constant). For the channel flow, $f_{i}=\delta_{i 1} f_{p}$, where $\delta_{i 1}$ is the Kronecker delta and $f_{p}$ is the pressure gradient imposed to achieve a desired mass flow rate. For the backward-facing step, $f_{i}=0$.

The governing equations (1) and (2) are discretized using second-order accurate central differences on a staggered grid and integrated with the fractional step method $[13,14]$. The time marching scheme for homogeneous isotropic turbulence is the third-order Runge-Kutta method for all terms, while for channel flow and a backward-facing step the third-order Runge-Kutta method is used for all the terms except the diffusion term in the wall-normal direction, for which the second-order Crank-Nicolson scheme is used. The Poisson solver for channel flow and homogeneous isotropic turbulence uses Fourier expansions in the streamwise and spanwise directions; for each wave-number pair the resulting tridiagonal matrix is inverted. For the backward-facing step a Fourier expansion is performed in the spanwise direction only and for each wave number a pentadiagonal matrix is inverted directly using cyclic reduction [15]. The code is parallelized using a message-passage 
interface and has been validated extensively for a variety of turbulent flows [16-18]. To represent the step in the backward-facing step simulation, an immersed boundary method with direct forcing was used [19].

\section{B. Subfilter-scale model}

In the ILSA model, the eddy viscosity is given by

$$
v_{\mathrm{SFS}}=\left(C_{k} L_{\mathrm{est}}\right)^{2}|\bar{S}|,
$$

where the integral length scale is estimated from

$$
L_{\text {est }}=\frac{\left\langle\mathcal{K}_{\text {res }}\right\rangle^{3 / 2}}{\left\langle\varepsilon_{\text {tot }}\right\rangle}
$$

here $\mathcal{K}_{\text {res }}=\bar{u}_{i}^{\prime} \bar{u}_{i}^{\prime} / 2$ (where $\bar{u}_{i}^{\prime}$ are the fluctuations of the resolved field) is the turbulent kinetic energy of the resolved scales and $\varepsilon_{\text {tot }}=2\left(v+v_{\mathrm{SFS}}\right) \bar{s}_{i j}^{\prime} \bar{s}_{i j}^{\prime}\left(\bar{s}_{i j}^{\prime}\right.$ is the fluctuating part of the resolved strain-rate tensor) is the total dissipation rate by the resolved $\left(\varepsilon_{v}=2 v \bar{s}_{i j}^{\prime} \bar{s}_{i j}^{\prime}\right)$ and subfilter eddies $\left(\varepsilon_{\mathrm{SFS}}=2 v_{\mathrm{SFS}} \bar{s}_{i j}^{\prime} \bar{s}_{i j}^{\prime}\right)$. Both $\mathcal{K}_{\text {res }}$ and $\varepsilon_{\text {tot }}$ are calculated locally and instantaneously. However, since $L_{\text {est }}$ is an integral quantity representing the average size of an ensemble of eddies, an averaging operation $\langle\cdots\rangle$ has been used in Eq. (5) to define it. In flows where directions of homogeneity exist, averaging can be performed over those directions; this results in an $L_{\text {est }}$ that varies in the nonhomogeneous directions (and time) only. Time averaging can also be used, and its application will be discussed in the next section.

Using (5) to define $L_{\text {est }}$, the filter width is

$$
\Delta=C_{\Delta} L_{\mathrm{est}}
$$

the exact value of the filter width, in practice, is not necessary, as the constant $C_{\Delta}$ is subsumed under $C_{k}$. Note that substituting (5) in Eq. (4) results in an implicit equation for $v_{\mathrm{SFS}}$ :

$$
v_{\mathrm{SFS}}=C_{k}^{2} \frac{\left\langle\mathcal{K}_{\mathrm{res}}\right\rangle^{3}}{\left\langle 2\left(v+v_{\mathrm{SFS}}\right) \bar{s}_{i j}^{\prime} \bar{s}_{i j}^{\prime}\right\rangle^{2}}|\bar{S}| .
$$

This issue is resolved by calculating the dissipation on the right-hand side at the previous time step.

To determine the parameter $C_{k}$ we use the following measure of SFS activity:

$$
s_{\tau}=\left[\frac{\left\langle\tau_{i j}^{a} \tau_{i j}^{a}\right\rangle}{\left\langle\left(\tau_{m n}^{a}+R_{m n}^{a}\right)\left(\tau_{m n}^{a}+R_{m n}^{a}\right)\right\rangle}\right]^{1 / 2},
$$

where $\tau_{i j}^{a}$ was defined in Eq. (3) and

$$
R_{i j}^{a}=\bar{u}_{i}^{\prime} \bar{u}_{j}^{\prime}-\frac{\delta_{i j}}{3} \bar{u}_{k}^{\prime} \bar{u}_{k}^{\prime}
$$

is the anisotropic parts of the resolved stresses.

A more intuitive measure of the SFS activity would be the SFS contribution to the turbulent kinetic energy

$$
s_{k}=\frac{\left\langle\mathcal{K}_{\mathrm{SFS}}\right\rangle}{\left\langle\mathcal{K}_{\text {tot }}\right\rangle}, \quad \mathcal{K}_{\text {tot }}=\frac{1}{2} u_{k}^{\prime} u_{k}^{\prime}=\overbrace{\frac{\bar{u}_{k}^{\prime} \bar{u}_{k}^{\prime}}{2}}^{\mathcal{K}_{\mathrm{res}}}+\overbrace{\frac{\tau_{k k}}{2}}^{\mathcal{K}_{\mathrm{SFS}}}
$$

this choice is however unfeasible within the present modeling assumption, due to the fact that the SFS turbulent kinetic energy (TKE) $\mathcal{K}_{\mathrm{SFS}}$ is unknown, since only the anisotropic part of the SFS stresses is modeled. In models in which this quantity is known (scale-similar or one-equation models, for instance), using $s_{k}$ might be more desirable. 


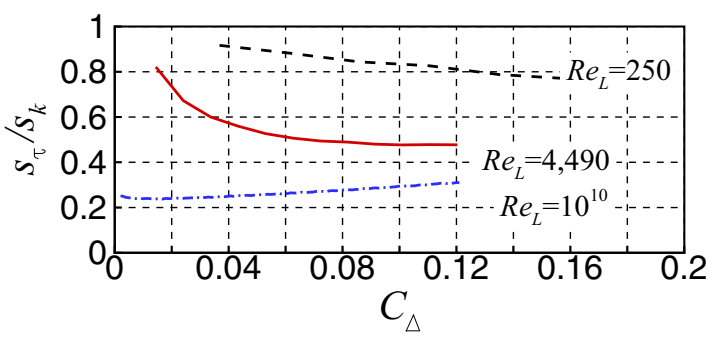

FIG. 1. Ratio $s_{\tau} / s_{k}$ against $C_{\Delta}$ at different Reynolds numbers for homogeneous isotropic turbulence: $\operatorname{Re}_{L}=250$ (black dashed line), $\operatorname{Re}_{L}=4490$ (red solid line), and $\operatorname{Re}_{L}=10^{10}$ (blue dash-dotted line) obtained from the model spectrum.

The relationship between $s_{\tau}$ and $s_{k}$ can be estimated, under the assumptions of high Reynolds number, eddy-viscosity hypothesis, and Gaussian behavior of fourth-order moments of the velocity field, by using a model spectrum [20]. The analysis is reported in the Appendix and its results are shown in Fig. 1. For large Reynolds numbers, both $s_{\tau}$ and $s_{k}$ become independent of the Reynolds number and vary with $C_{\Delta}$ only and the ratio $s_{\tau} / s_{k}$ has a value of the order of $0.25-0.35$ for $0.025<C_{\Delta}<0.12$ (a realistic range, corresponding to a filter width that is $1 / 40$ to $1 / 8$ of the integral length scale). This value is only an indication, since in more complex applications the Reynolds number is finite and other effects (shear, for instance) are important, and $s_{\tau} / s_{k}$ takes different values, as will be shown.

We also carried out two DNSs of forced homogeneous isotropic turbulence at $\operatorname{Re}_{L}=250$ and 4490 (based on $\left\langle\mathcal{K}_{\text {tot }}\right\rangle^{1 / 2}$ and $L=\left\langle\mathcal{K}_{\text {tot }}\right\rangle^{3 / 2} /\left\langle\varepsilon_{\text {tot }}\right\rangle$ ). The DNS data were obtained using $256 \times 256 \times 256$ and $512 \times 512 \times 512$ grid points (at the low and high Reynolds numbers, respectively) to discretize a triply periodic box with dimensions $2 \pi$ in each direction. The results compared well with data in the literature [12] (not shown). The cutoff wave number was defined as $K_{\mathrm{c}}=\pi / C_{\Delta} L$, the DNS data were filtered using a three-dimensional box filter of width $\Delta=C_{\Delta} L$, and the ratio $s_{\tau} / s_{k}$ was computed. The lower bound for $C_{\Delta}$ is restricted by the grid resolution, with a filter width encompassing three cells $\left(\Delta_{\min }=3 h\right)$, and its upper bound is assigned based on a filter width that yields $s_{k} \simeq 0.2-0.25$, the limit for a well-resolved LES. The results are also shown in Fig. 1 . At low Reynolds number $\left(\operatorname{Re}_{L}=250\right), s_{\tau}$ and $s_{k}$ are close to each other and $s_{\tau} / s_{k} \simeq 1.0$. As the Reynolds number is increased to $\operatorname{Re}_{L}=4490, s_{\tau}$ becomes a fraction of $s_{k}$ with $s_{\tau} / s_{k} \simeq 0.5$ at large enough $C_{\Delta}$.

We also calculated the ratio $s_{\tau} / s_{k}$ for DNS of channel flow at $\operatorname{Re}_{\tau}=395$ (based on channel half height $\delta$ and friction velocity $u_{\tau}$ ). We performed a DNS using $512 \times 256 \times 512$ grid points to discretize a domain of size $8 \delta \times 2 \delta \times 4 \delta$ and first- and second-order statistics compared well with the data in the literature [21]. Filtering was carried out at each $y$ level using a two-dimensional square filter of width $\Delta=n \Delta x$, where $n=3,5,7$ and $\Delta x$ is the streamwise grid size. Since the integral scale varies across the channel, so does $\operatorname{Re}_{L}=L\left\langle\mathcal{K}_{\text {tot }}\right\rangle^{1 / 2} / v$ (where $\left\langle\mathcal{K}_{\text {tot }}\right\rangle$ is the plane-averaged TKE across the channel); $\operatorname{Re}_{L}$ [Fig. 2(a)] is comparable to the low-Reynolds-number homogeneous isotropic turbulence simulation. Also $C_{\Delta}=\Delta / L$ changes [Fig. 2(b)]; near the wall, in the sheardominated region, it reaches values close to one, indicating that even the integral scale of turbulence is only marginally resolved. In this situation, the role of the SFS model becomes critical, although many of the assumptions on which SFS models rest (the filter width is in the inertial range and the unresolved scales carry most of the energy) are not strictly satisfied. Figure 2(c) shows the profiles of the ratio $s_{\tau} / s_{k}$. Away from the wall $(y \geqslant 0.2)$ where shear is small, the results are consistent with those of the low-Reynolds-number homogeneous isotropic turbulence simulation, while near the wall, where the shear stress becomes large, this ratio exceeds one.

To summarize, we find that $s_{\tau}$ is a legitimate surrogate for $s_{k}$, the fraction of TKE provided by the unresolved scales. In the absence of shear, in the limit of large Reynolds number, and with the 

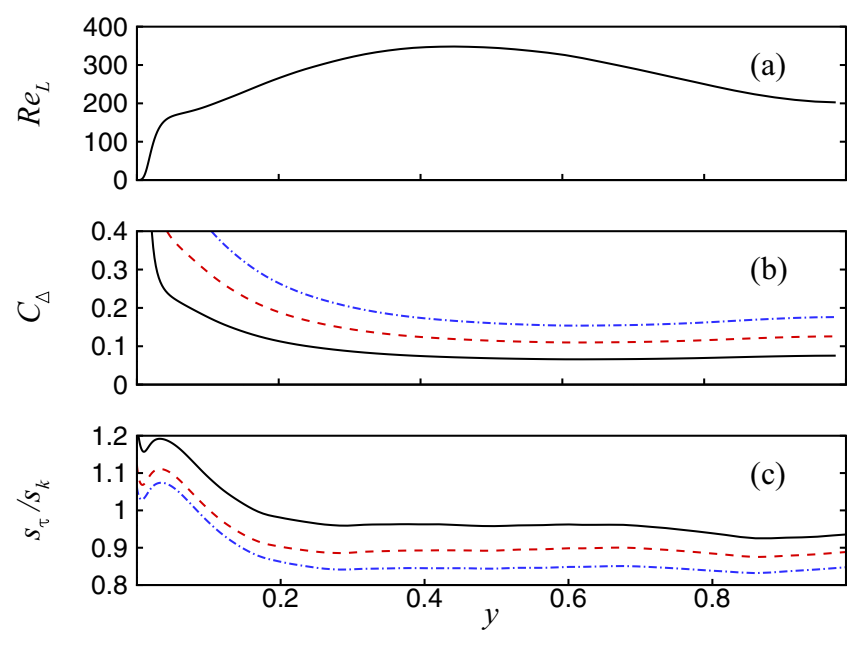

FIG. 2. Channel flow at $\operatorname{Re}_{\tau}=395$ for (a) $\operatorname{Re}_{L}=L\left\langle\mathcal{K}_{\text {tot }}\right\rangle^{1 / 2} / \nu$, (b) $C_{\Delta}$, and (c) $s_{\tau} / s_{k}$ with $\Delta=3 \Delta x$ (black solid line), $\Delta=5 \Delta x$ (red dashed line), and $\Delta=7 \Delta x$ (blue dash-dotted line).

filter width in the inertial region of the spectrum, $s_{k} \simeq 4 s_{\tau}$, whereas in high-shear regions $s_{k} \simeq s_{\tau}$. If we require that the resolved scales contribute more than $80 \%$ of the TKE (a reasonable value for a well-resolved LES), we should expect $s_{\tau}<0.05$. This value is consistent with the results of our numerical tests, as will be shown later.

The second modification made to the ILSA model consists in the localization of the model coefficient. As discussed above, appropriate LES resolution is satisfied by maintaining $s_{\tau}$ below a threshold value. However, proper quantification of SFS activity relies on scale homogeneity and thus consistency in the shape of the energy spectrum. In a channel flow, for instance, scale homogeneity exists in each plane parallel to the wall, while in a complex flow problem, time in a Lagrangian fashion [22] can be considered as the scale-homogeneous dimension. In the original formulation, the SFS activity was averaged over the entire computational domain and time and the model coefficient $C_{k}$ derived from this procedure was everywhere constant. Due to the global assignment of SFS activity, the original formulation was called the global ILSA. When the global ILSA is applied to channel flow, since the enforced SFS activity is an integral quantity, the SFS activity values that result from each wall-parallel plane differ from each other and are either smaller or larger than the global enforced value [7]. A more general target of SFS activity could be averaged locally, over homogeneous directions only, and/or over time. This formulation is named the local ILSA; local enforcement of the desired SFS activity requires $C_{k}$ to be computed locally as well. The local attribute changes its implication depending on the choice of averaging; if averaging is carried out over $x z$ planes, for instance, then $C_{k}$ is local with respect to the $y$ coordinate and time, whereas for averaging over time the model yields local $C_{k}$ as a function of $x, y, z$, within certain limits.

Note that calculating $C_{k}$ instantaneously and thus removing the averaging operation from (8) and (5) is not consistent with the model assumptions that require the estimation of the integral length scale, which is an average quantity; it will be shown in Sec. III that instantaneous calculation of $C_{k}$ leads to an error in the integral scale. Performing integration over the homogeneous directions of the flow (if any) and over time appears sensible. In the flows examined in this paper, the plane channel and backward-facing step, homogeneous directions exist: $x$ and $z$ for the channel and $z$ only for the backward-facing step. Averaging can also be performed in time, over a window whose length should be proportional to the integral time scale, either at a fixed point in Eulerian space or following a Lagrangian approach similar to that of Meneveau et al. [23]. Most of the results shown in the following were obtained using spatial averaging over homogeneous directions. In Sec. III C we 

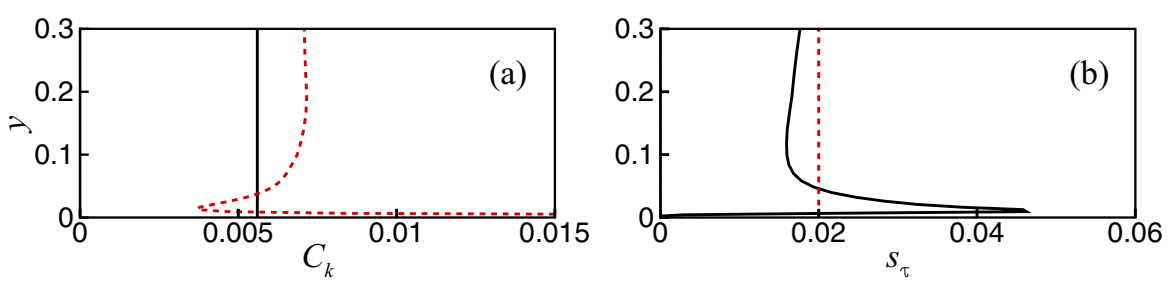

FIG. 3. Model coefficient and SFS activity with local and global averaging: (a) model coefficient $C_{k}$ and (b) SFS activity measure $s_{\tau}$ for global averaging (black solid line) and local averaging (red dashed line) with channel flow at $\operatorname{Re}_{\tau}=1000$ with $48 \times 64 \times 48$ grid points.

discuss temporal averaging and demonstrate the insensitivity of the model to the type of averaging (spatial or temporal).

Figure 3 shows the difference between the global and local ILSA approaches observed from the calculation of a plane channel flow at $\operatorname{Re}_{\tau}=1000$ to be discussed in the next section. With the global formulation, the use of a constant $C_{k}$ over the entire channel resulted in a significantly larger contribution of the SFS to the transport in the near-wall region. A constant $C_{k}$ also implies that the ratio of the filter width to the local integral scale is constant throughout the channel; the size of the turbulent eddies and the shape of the spectrum, however, are significantly different between the near-wall and the outer regions of the flow and one would expect that the filter width would need to account for this. Using a local formulation (averaging is only performed in the $x z$ plane, in this case) forces the SFS activity to have the assigned value at each $y$ level, resulting in a uniform distribution of $s_{\tau}$, and a nonuniform distribution of $C_{k}$ across the channel. This results in a physically consistent behavior of $C_{k}$, which becomes smaller as the wall is approached. In the viscous sublayer $\left(y^{+}<10\right)$ the coefficient increases: This is due to the fact that the grid is not fine enough to resolve the smaller eddies present in that region, so all the transport becomes due to unresolved eddies; this issue will be discussed in more detail later.

The present approach has an additional benefit: the fact that $C_{k}$ can be calculated directly, without the a priori calculations used in Ref. [7]. If (3) and (4) are used in Eq. (8), a quadratic equation results for $C_{k}$ :

$$
\mathcal{X}_{1}\left[1-\left(1 / s_{\tau}\right)^{2}\right] C_{k}^{4}-\mathcal{X}_{2} C_{k}^{2}+\mathcal{X}_{3}=0
$$

where

$$
\mathcal{X}_{1}=\left\langle 2 L_{\text {est }}^{4}|\bar{S}|^{4}\right\rangle, \quad \mathcal{X}_{2}=\left\langle 4 L_{\text {est }}^{2}|\bar{S}| \bar{S}_{i j} R_{i j}^{a}\right\rangle, \quad \mathcal{X}_{3}=\left\langle R_{m n}^{a} R_{m n}^{a}\right\rangle
$$

Equation (11) can be solved, at each time step, for each $y=$ const plane (for the channel) or at each point in the $x y$ plane (for the backward-facing step). The only positive root is used in the model.

\section{RESULTS}

The localized ILSA model was applied to plane channel flow at fairly high Reynolds numbers $\left(\operatorname{Re}_{\tau}=1000\right.$ and 2000, where the Reynolds number is based on the friction velocity $u_{\tau}$ and the channel half height $\delta$ ) and to a BFS at $\operatorname{Re}_{c}=28000$ (based on the mean centerline velocity of the inflow channel $U_{c}$ and the step height $h_{s}$ ). In addition to the comparison with DNS and experimental data, we will stress the response of the model to grid refinement and its behavior in the near-wall region. We will also carry out a comparison between local and global ILSAs and the dynamic eddy-viscosity model [24].

\section{A. Plane channel flow}

The local ILSA was applied to study channel flow at $\operatorname{Re}_{\tau}=1000$ and 2000 . The domain size is $6 \delta \times 2 \delta \times 3 \delta$ with a periodic boundary condition applied in the streamwise and spanwise directions 
TABLE I. Summary of plane-channel flow simulations.

\begin{tabular}{|c|c|c|}
\hline Grid & & Model \\
\hline \multicolumn{3}{|c|}{$\operatorname{Re}_{\tau}=1000$} \\
\hline $48 \times 65 \times 48$ & & local ILSA, $s_{\tau}=0.020$ \\
\hline $48 \times 65 \times 48$ & & local ILSA, $s_{\tau}=0.022$ \\
\hline $64 \times 97 \times 64$ & & local ILSA, $s_{\tau}=0.020$ \\
\hline $64 \times 97 \times 64$ & & local ILSA, $s_{\tau}=0.022$ \\
\hline $128 \times 129 \times 128$ & & local ILSA, $s_{\tau}=0.020$ \\
\hline $128 \times 129 \times 128$ & & local ILSA, $s_{\tau}=0.022$ \\
\hline $192 \times 193 \times 192$ & & local ILSA, $s_{\tau}=0.022$ \\
\hline $128 \times 129 \times 128$ & & local ILSA, $s_{\tau}=0.010$ \\
\hline $128 \times 129 \times 128$ & & local ILSA, $s_{\tau}=0.030$ \\
\hline $48 \times 65 \times 48$ & & global ILSA, $s_{\tau}=0.020$ \\
\hline $64 \times 97 \times 64$ & & global ILSA, $s_{\tau}=0.020$ \\
\hline $128 \times 129 \times 128$ & & global ILSA, $s_{\tau}=0.020$ \\
\hline $48 \times 65 \times 48$ & & dynamic model \\
\hline $64 \times 97 \times 64$ & & dynamic model \\
\hline $128 \times 129 \times 128$ & & dynamic model \\
\hline \multicolumn{3}{|c|}{$\operatorname{Re}_{\tau}=2000$} \\
\hline $64 \times 97 \times 64$ & & local ILSA, $s_{\tau}=0.022$ \\
\hline $128 \times 129 \times 128$ & & local ILSA, $s_{\tau}=0.022$ \\
\hline $192 \times 193 \times 192$ & & local ILSA, $s_{\tau}=0.022$ \\
\hline $256 \times 257 \times 256$ & & local ILSA, $s_{\tau}=0.022$ \\
\hline $192 \times 193 \times 192$ & & local ILSA, $s_{\tau}=0.010$ \\
\hline $192 \times 193 \times 192$ & & local ILSA, $s_{\tau}=0.030$ \\
\hline $192 \times 193 \times 192$ & & dynamic model \\
\hline
\end{tabular}

and a no-slip condition at the top and bottom boundaries. Simulations were carried out with both the local and the global ILSA models (with various target values for the SFS activity measure) as well as with the plane-averaged dynamic model to create a well-known point of comparison (see Table I).

Now we examine the effect of the SFS activity threshold. In Fig. 4 we compare the results obtained with $s_{\tau}=0.01,0.022$, and 0.03 . The grid was chosen based on the grid convergence study to be discussed momentarily. Given the expected relationship between $s_{\tau}$ and $s_{k}$, the percentage of TKE in the unresolved scales, these values would correspond to a well-resolved simulation, with between $88 \%$ (in the channel center) and 95\% (near the wall) of the energy being resolved. The agreement with the DNS data is generally very good. For $s_{\tau}=0.03$ the model is more dissipative, the buffer layer becomes thicker, and the logarithmic layer in the velocity profile is shifted upward. Conversely, when the SFSs do not contribute enough, the wall stress is overpredicted and the logarithmic layer is shifted downward [Figs. 4(a) and 4(c)]. The errors in the prediction of the skin-friction coefficient are $-10 \%$ and $-2 \%$ for $s_{\tau}=0.03$ (at the lower and higher $\operatorname{Re}_{\tau}$, respectively) and $2 \%$ for $s_{\tau}=0.01$. Both the peak value of streamwise root-mean-square ( $\mathrm{rms}$ ) fluctuations and its location are predicted well in all cases. The thickening of the buffer layer, however, can be observed by zooming into the region near the peak streamwise rms fluctuations [the insets in Figs. 4(b) and 4(d)], which show a sharper peak for lower values of $s_{\tau}$.

Another measure of SFS activity is the percentage of Reynolds shear stress supported by the unresolved scales, which can be defined as

$$
s_{u v}=\frac{\left\langle\tau_{u v}^{a}\right\rangle}{\left\langle\bar{u}^{\prime} \bar{v}^{\prime}+\tau_{u v}^{a}\right\rangle} .
$$



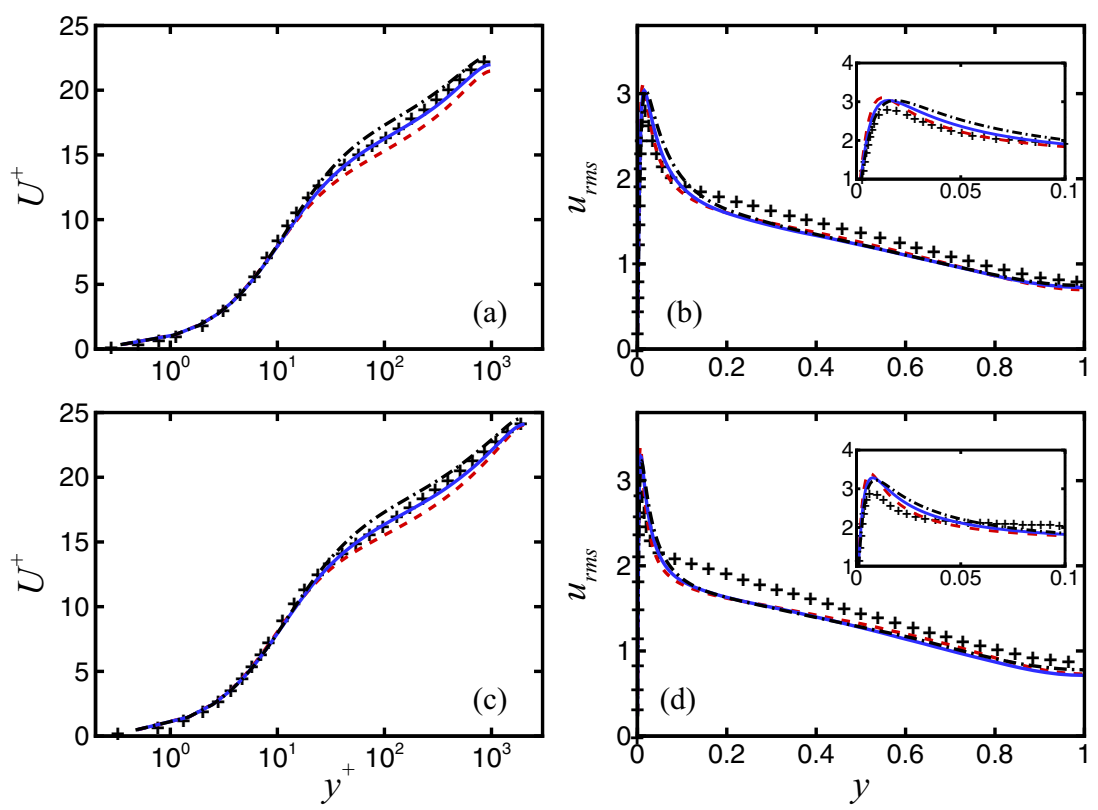

FIG. 4. Mean velocity and streamwise root-mean-square velocity fluctuations profiles at (a) and (b) $\operatorname{Re}_{\tau}=$ 1000 with $128 \times 129 \times 128$ grid points and (c) and (d) $\operatorname{Re}_{\tau}=2000$ with $192 \times 193 \times 192$ grid points with $s_{\tau}=0.010$ (red dashed line), $s_{\tau}=0.022$ (blue solid line), $s_{\tau}=0.030$ (black dash-dotted line), and DNS [25] (pluses).

Although $s_{u v}$ is not generally applicable because it is not frame invariant, it yields useful information, in this case, on the level of resolution implied by the three chosen values of $s_{\tau}$.

Figure 5 compares the value of $s_{u v}$ for the three simulations shown above with the analogous quantity obtained from a simulation with the dynamic eddy-viscosity model [24]. As expected, the SFS stress increases as $s_{\tau}$ increases. Outside the wall layer, $s_{u v} \simeq s_{\tau}$; in the viscous sublayer, with the ILSA model, the SFSs contribute increasingly to the momentum flux and the wall value of $s_{u v}$ approaches unity. The behavior of the ILSA model is due to the fact that near the wall the integral scale increases linearly, while the grid is refined only in the wall-normal direction; thus, the unresolved scales should contribute most (or all) of the momentum transport. We notice, however, that the thickening of the buffer layer mentioned above is associated with large values of $s_{u v}$ that persist through the buffer layer. By contrast, the shear stress predicted by the dynamic model is much lower and near the wall the SFS contribution to the momentum flux approaches a smaller value, close to 0.2 , because the filter width is proportional to the grid size, and the eddy viscosity decreases too rapidly.
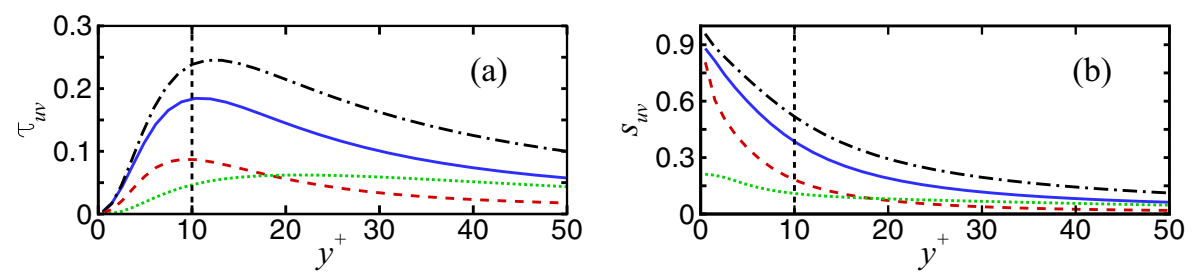

FIG. 5. Contribution of SFSs to the streamwise momentum flux in channel flow at $\operatorname{Re}_{\tau}=2000$ with $192 \times 193 \times 192$ grid points for (a) $\tau_{u v}$ and (b) $s_{u v}$ with $s_{\tau}=0.010$ (red dashed line), $s_{\tau}=0.022$ (blue solid line), $s_{\tau}=0.030$ (black dash-dotted line), and dynamic model [24] (green dotted line). 

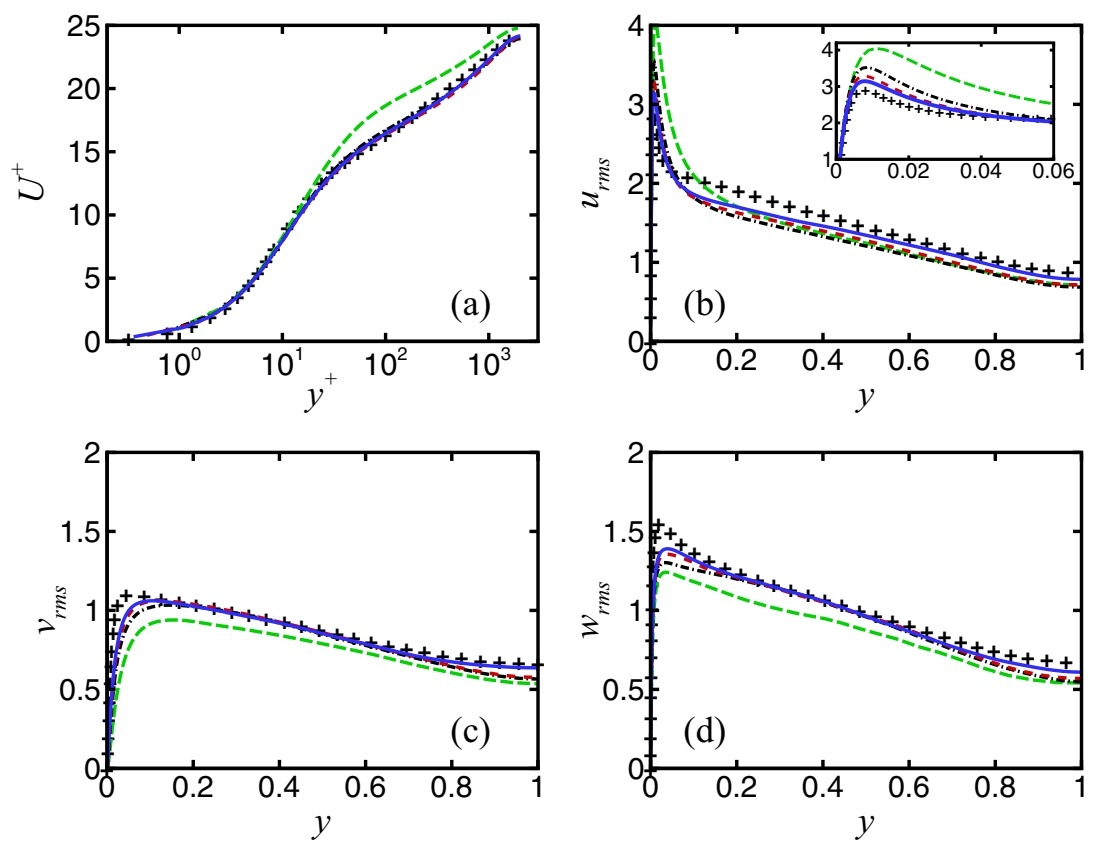

FIG. 6. Turbulence statistics at $\operatorname{Re}_{\tau}=2000$ using the local ILSA and $s_{\tau}=0.022$ : (a) mean velocity, (b) $u_{\mathrm{rms}}$, (c) $v_{\mathrm{rms}}$, and (d) $w_{\mathrm{rms}}$, all normalized by $u_{\tau}$, with $64 \times 97 \times 64$ grid points (green long-dashed line), $128 \times 129 \times 128$ grid points (black dash-dotted line), $192 \times 193 \times 192$ grid points (red short-dashed line), $256 \times 257 \times 256$ grid points (blue solid line), and DNS [25] (pluses).

We next consider the grid resolution requirements. We performed simulations for both Reynolds numbers using the value of $s_{\tau}=0.022$ that was found to yield the most accurate results. Figure 6 shows the results at $\operatorname{Re}_{\tau}=2000$. Both the mean and rms velocities reach grid convergence. For the mean velocity $128 \times 129 \times 128$ grid points are required, while the rms velocities need $192 \times$ $193 \times 192$ grid points to become grid independent. At $\operatorname{Re}_{\tau}=1000$ earlier grid convergence was reached for the mean using $64 \times 97 \times 64$ and for the rms fluctuations using $128 \times 129 \times 128$ points. Accurate prediction of the mean velocity on the coarse grid using the local ILSA was also observed in the global ILSA [7]. This is an additional advantage of these two models, which will be explained momentarily through further study of their near-wall behavior.

The variation across the channel of the eddy viscosity and $C_{k}$ is shown in Fig. 7. At $\operatorname{Re}_{\tau}=1000$ the eddy viscosity and $C_{k}$ become grid independent when $128 \times 129 \times 128$ grid points are used, similarly to the second-order statistics. In the local ILSA, as shown in Fig. 7(b), the eddy viscosity near the wall is proportional to $y^{2}$, not far from the required $y^{3}$ behavior. This is an improvement over the global ILSA model, in which near the wall the eddy viscosity is proportional to $y^{6}$ when $v_{\mathrm{SFS}} \ll v$ and to $y^{2}$ only if $v_{\mathrm{SFS}} \gg v$. The difference between the two models is due to the fact that in global ILSA $C_{k}$ is spatially constant and $\nu_{\mathrm{SFS}} \propto L_{\text {est }}^{2}$. In the localized model $C_{k}$ is allowed to vary. To explain the near-wall behavior, (8) can be rewritten in the form

$$
\left(1-1 / s_{\tau}^{2}\right)\left\langle\tau_{i j}^{a} \tau_{i j}^{a}\right\rangle-2 / s_{\tau}^{2}\left\langle R_{i j}^{a} \tau_{i j}^{a}\right\rangle-\left\langle R_{i j}^{a} R_{i j}^{a}\right\rangle=0
$$

substituting (3) and (9) for $\tau_{i j}^{a}$ and $R_{i j}^{a}$, expanding $\bar{u}_{i}$ as a Taylor series of $y$, and retaining only the leading-order terms, we obtain

$$
a v_{\mathrm{SFS}}^{2}+b v_{\mathrm{SFS}} y^{2}+c y^{4}=0
$$

where $a, b$, and $c$ are independent of $y$. The above equation is only satisfied if $\nu_{\mathrm{SFS}} \propto y^{2}$. The near-wall behavior of $v_{\mathrm{SFS}}$ implies that $C_{k} \propto y^{-2}$ at low Reynolds numbers; this can also be observed 

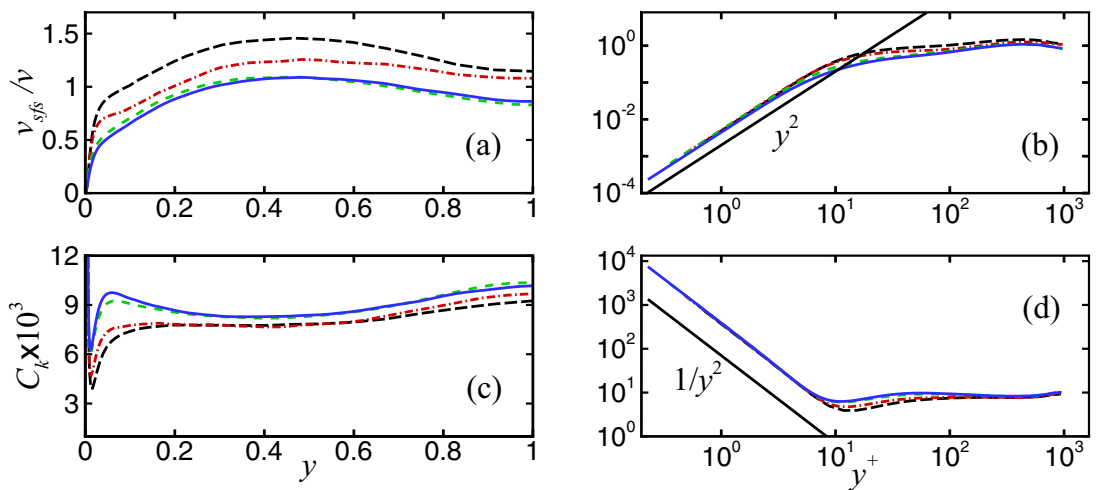

FIG. 7. Profiles of (a) and (b) normalized SFS eddy viscosity $v_{\mathrm{SFS}} / v$ and (c) and (d) $C_{k}$ at $\operatorname{Re}_{\tau}=1000$ with $48 \times 65 \times 48$ (black long-dashed line), $64 \times 97 \times 64$ (red dash-dotted line), $128 \times 129 \times 128$ (green short-dashed line), and $192 \times 193 \times 192$ (blue solid line) grid points.

in Fig. 7(d). We conclude by observing that formulating $s_{\tau}$ locally not only gives better control of the SFS activity, but also improves the near-wall behavior of the eddy viscosity, compared to the global ILSA.

The local and global ILSA models were also compared with the dynamic eddy-viscosity model [24]. Figures 8 and 9 show the mean and rms velocity profiles at different grid resolutions, respectively. We have also added coarse DNS simulations without an explicit SFS term to quantify the explicit model contribution.

Piomelli et al. [7] observed higher accuracy in the global ILSA compared to the dynamic model on coarse grids; the local ILSA preserves this property, in particular in the mean velocity. The better agreement of the ILSA model on a coarse grid is due to its near-wall behavior, shown in Figs. 10(a) and 10 (b). Near the wall the eddy viscosity increases more rapidly compared to the dynamic model so that, on the coarse grid, the eddy viscosity in the buffer layer is 4-8 times larger than the one produced by the dynamic model. Such high model activity compensates for the lack of momentum

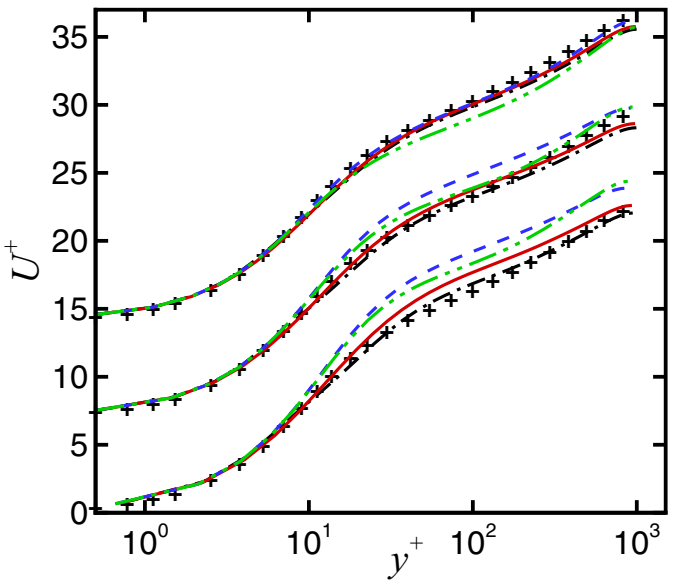

FIG. 8. Mean velocity profiles in inner units at $\mathrm{Re}_{\tau}=1000$ for DNS [25] (pluses), the dynamic model [24] (blue dashed line), the local ILSA with $s_{\tau}=0.02$ (red solid line), the global ILSA with $s_{\tau}=0.02$ (black dash-dotted line), and no model (green dash-double-dotted line). The bottom curves show $48 \times 65 \times 48$ grid points, the middle curves $64 \times 97 \times 64$ grid points, and the top curves $128 \times 129 \times 128$ grid points. 

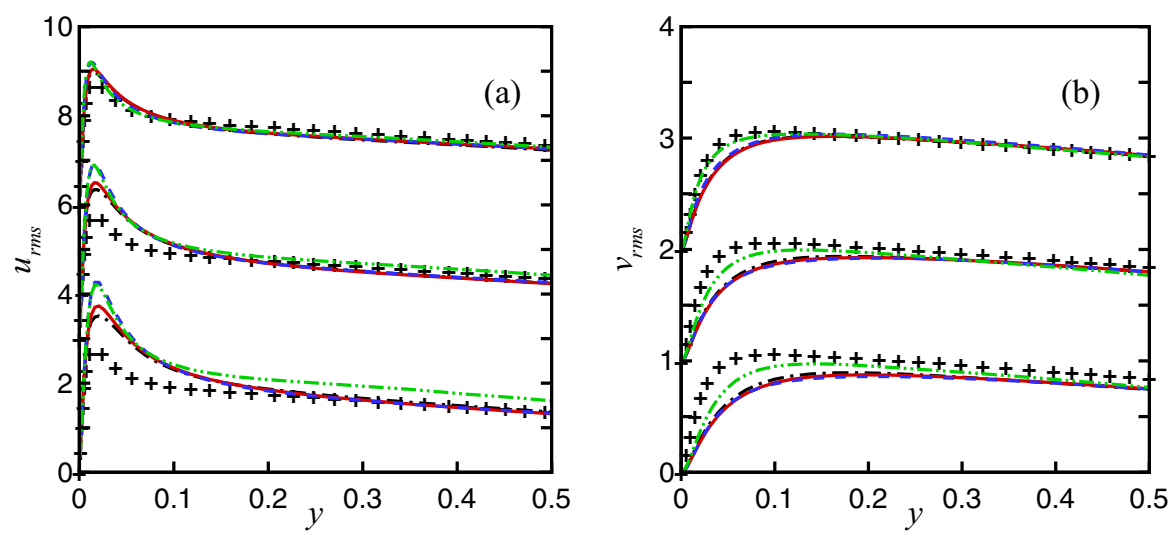

FIG. 9. Profiles of (a) $u_{\mathrm{rms}}$ and (b) $v_{\mathrm{rms}}$ at $\operatorname{Re}_{\tau}=1000$. Legends and grid resolutions are consistent with Fig. 8. For clarity the data are shown up to $y=0.5$.

transport due to underresolved eddies on a coarse grid. It is easy to show that near the wall $s_{u v}$ is proportional to $y^{-1}$ for the local ILSA model; $y^{-1}$ or $y^{0}$ for the global ILSA model, at high or low Re, respectively; and $y^{0}$ for the dynamic model. These behaviors can be observed in Figs. 10(c) and $10(\mathrm{~d})$.

The effect of the model contribution to transport can also be observed from Figs. 8 and 9. On the coarse and intermediate grids the mean velocity and streamwise fluctuations are predicted more accurately when the model is used; the mean velocity is more sensitive than the rms fluctuations to the model. Note that the results are grid converged on the intermediate grid and the fine one is excessively refined. When the fine grid is used, the rms fluctuations obtained by the coarse DNS are in very close agreement with the LES, while the mean velocity (and the wall stress) are predicted less accurately. Also, both simulations that use SFS models have a more reliable grid-convergence behavior than the coarse DNS. The coarse DNS in fact gives reasonably good agreement with the data at $64 \times 97 \times 64$ grid points, but as the resolution is doubled, error appears in the wall shear stress and hence in the logarithmic region intercept. Such behavior was previously addressed in the literature [26,27]; Meyers and Sagaut [26], using an energy-conserving code, observed that underresolved DNS predicts the wall friction and the mean velocity accurately at a certain resolution,
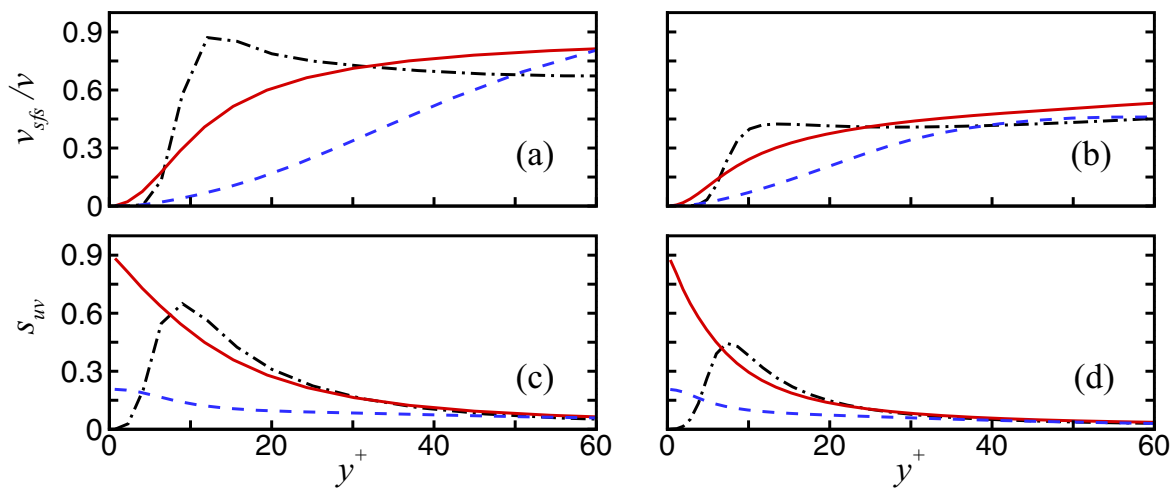

FIG. 10. Plots of SFS parameters at $\operatorname{Re}_{\tau}=1000$ for the dynamic model [24] (dashed blue line), the local ILSA with $s_{\tau}=0.02$ (red solid line), and the global ILSA with $s_{\tau}=0.02$ (black dash-dotted line) with (a) and (c) $48 \times 65 \times 48$ and (b) and (d) $128 \times 129 \times 128$ grid points for (a) and (b) the ratio of subfilter eddy viscosity over kinematic viscosity and (c) and (d) $s_{u v}$. 
TABLE II. Simulation cost for channel flow at $\operatorname{Re}_{\tau}=1000$ using $128 \times 129 \times 128$ grid points.

\begin{tabular}{lccc}
\hline \hline Model & Average time step & CPU per time step (s) & Total CPU (h) \\
\hline Smagorinsky & 0.0055 & 1.065 & 10.55 \\
global ILSA, $s_{\tau}=0.02$ & 0.0056 & 1.142 & 11.1 \\
local ILSA, $s_{\tau}=0.02$ & 0.0056 & 1.151 & 11.19 \\
dynamic [24] & 0.0056 & 1.374 & 13.35 \\
\hline \hline
\end{tabular}

but this accuracy may disappear once the grid is refined. This illustrates complex error behavior at coarse resolution, which is not in the fully asymptotic regime yet.

The computational cost of the models is summarized in Table II; all the simulations were run at $\operatorname{Re}_{\tau}=1000$ using $128 \times 129 \times 128$ grid points for $10 \delta / u_{\tau}$ on eight $2.52-\mathrm{GHz}$ Sparc64 VII processors. The difference between the global and local ILSA models is marginal, while the dynamic model [24] is approximately $20 \%$ more expensive, due to the extra filtering operations. The cost of the ILSA models is only $6 \%$ higher than that of the Smagorinsky model.

\section{B. Backward-facing step}

The BFS is a canonical benchmark test case for turbulence models; it includes flow separation and reattachment, recirculation, and flow acceleration. To assess the local ILSA and its accuracy compared to other models in a geometry more complex than the plane channel tested so far we performed calculations of the backward-facing step in conditions matching the experiment of Vogel and Eaton [28]. The Reynolds number is $\operatorname{Re}_{c}=28000$, based on the mean centerline velocity of the inflow channel $U_{c}$ and the step height $h_{s}$. Figure 11 shows the domain size and grid arrangement; the origin is placed at the bottom edge of the step. The height of the inflow channel is $4 h_{s}$ and the expansion ratio is 1.25 ; the spanwise width is $W=3 h_{s}$. To generate a fully developed turbulent channel inflow, the velocity field at $x=-5 h_{s}$ was recycled to the inlet plane at $x=-32 h_{s}$; thus our inflow length is $L_{\mathrm{ch}}=27 h_{s}, 13.5$ times the inflow channel half height. The mean and rms velocity profiles in the inlet channel were in agreement with those obtained from simulations of periodic channel flow. At the outlet, a convective outflow boundary condition [29] was used. The length of the expanded duct is $L_{\text {out }}=20 h_{s}$, which was also used in previous LES simulations [17,30]. To ensure the adequacy of $L_{\text {out }}$, a longer domain with $L_{\text {out }}=30 h_{s}$ was also used; the flow statistics at $x=19 h_{s}$ showed very little difference.

Figure 11 highlights the clustering of grid points at the step edge and near the walls. The models were tested on three grids, summarized in Table III: coarse $(256 \times 100 \times 64)$, intermediate $(384 \times$ $150 \times 96)$, and fine $(512 \times 200 \times 128)$. Following Yu and Moin [30], wall units were calculated using the friction velocity at the outlet. Also, since our domain of interest is $x / h_{s}<10$, the adequacy of the streamwise grid size is assessed within this range only. The intermediate resolution

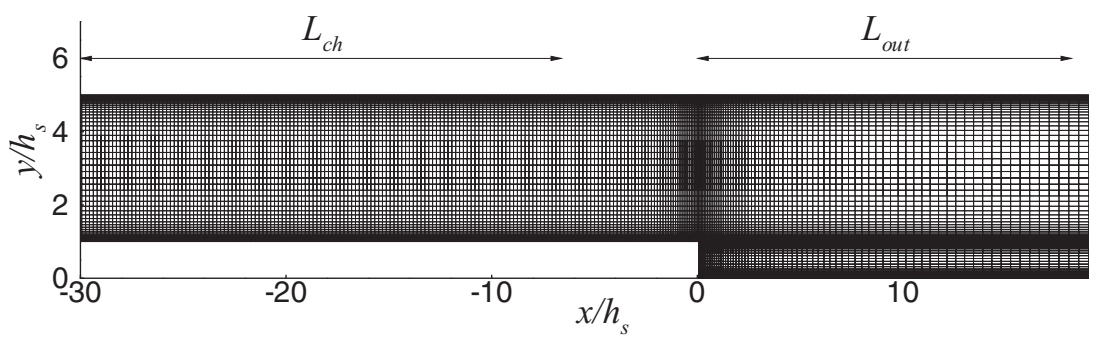

FIG. 11. Grid size and arrangement for the backward-facing step calculations using the coarse grid $256 \times$ $100 \times 64$. Grid size distributions $\Delta x / h_{s}$ and $\Delta y / h_{s}$ are normalized by the step height $h_{s}$. 
TABLE III. Summary of grid resolutions for a backward-facing step. Grid sizes are normalized by the friction velocity at the outlet $u_{\tau}\left(x / h_{s}=20\right)$.

\begin{tabular}{lcccr}
\hline \hline Grid level & $N_{x} \times N_{y} \times N_{z}$ & $\Delta x^{+}\left(x / h_{s}<10\right)$ & $\Delta y^{+}$ & $\Delta z^{+}$ \\
\hline coarse & $256 \times 100 \times 64$ & $42.8-244.2$ & $0.9-122.3$ & 33.5 \\
intermediate & $384 \times 150 \times 96$ & $28.7-163.4$ & $0.6-80.4$ & 21.5 \\
fine & $512 \times 200 \times 128$ & $19.3-109.1$ & $0.4-54.2$ & 14.3 \\
\hline \hline
\end{tabular}

was adopted in previous studies of this problem [17,30]. Here we also consider a coarser and a finer level for a more extensive comparison. The local ILSA model used $s_{\tau}=0.022$ and the averaging was performed in the spanwise direction only.

The accuracy of the local ILSA was assessed through comparison with the Lagrangian dynamic model [23] and the experimental data [28]; the mean velocity is shown in Fig. 12 and the streamwise turbulence intensity in Fig. 13. The ILSA model is slightly more accurate than the dynamic model on the coarsest grid in the mean velocity (after the flow reattachment) and Reynolds shear stress (within the circulation bubble). We also compared the Reynolds shear stress in Fig. 14 (note that the SFS shear stress $\tau_{12}$ was added to the resolved one $\left\langle\bar{u}^{\prime} \bar{v}^{\prime}\right\rangle$ ); experimental data were not available for the Reynolds shear stress at the locations considered.

We also performed calculations with no model. On the coarse and grid-converged meshes, the coarse DNS results in significant errors in the mean velocity, especially in the prediction of the reattachment point (Fig. 12). Even on the fine mesh some differences with the LES and experimental data persist. The error in the rms fluctuations is similar, while that in the Reynolds shear stress (Fig. 14) is higher when no model is used.
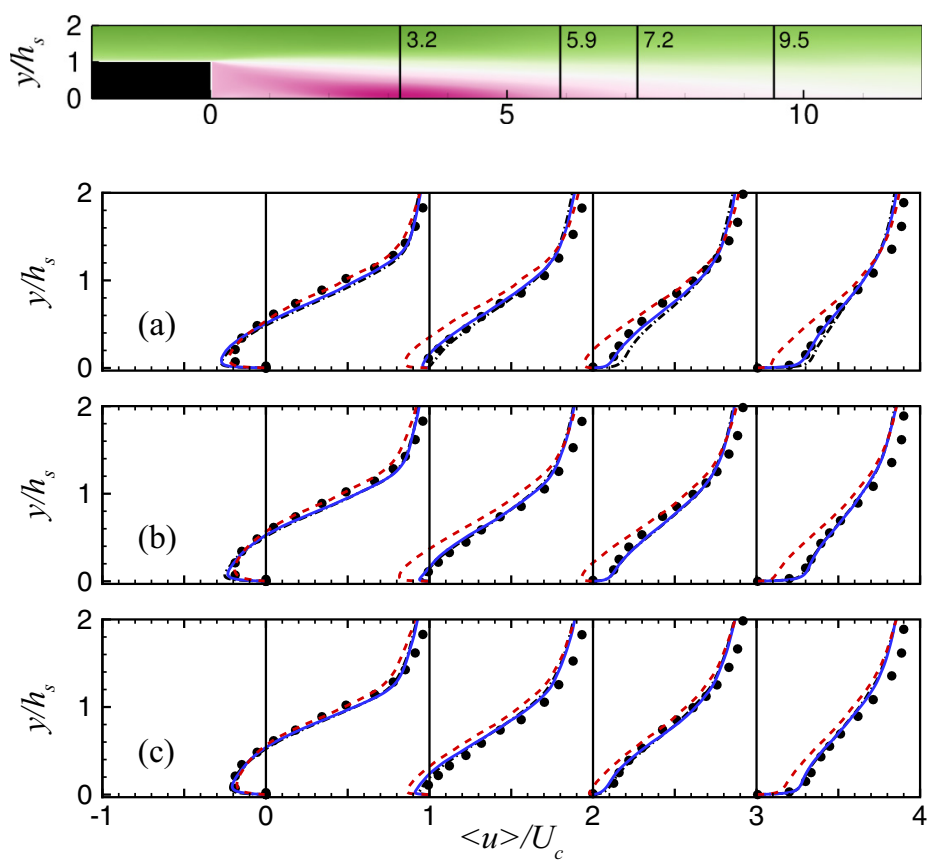

FIG. 12. Mean velocity normalized by the centerline velocity at the inlet duct at the locations specified downstream of the step with (a) a coarse $256 \times 100 \times 64$ grid, (b) an intermediate $384 \times 150 \times 96$ grid, and (c) a fine $512 \times 200 \times 128$ grid for the local ILSA with $s_{\tau}=0.022$ (solid blue line), no model (red dashed line), the Lagrangian dynamic model [23] (black dash-dotted line), and experiment [28] (closed circles). 

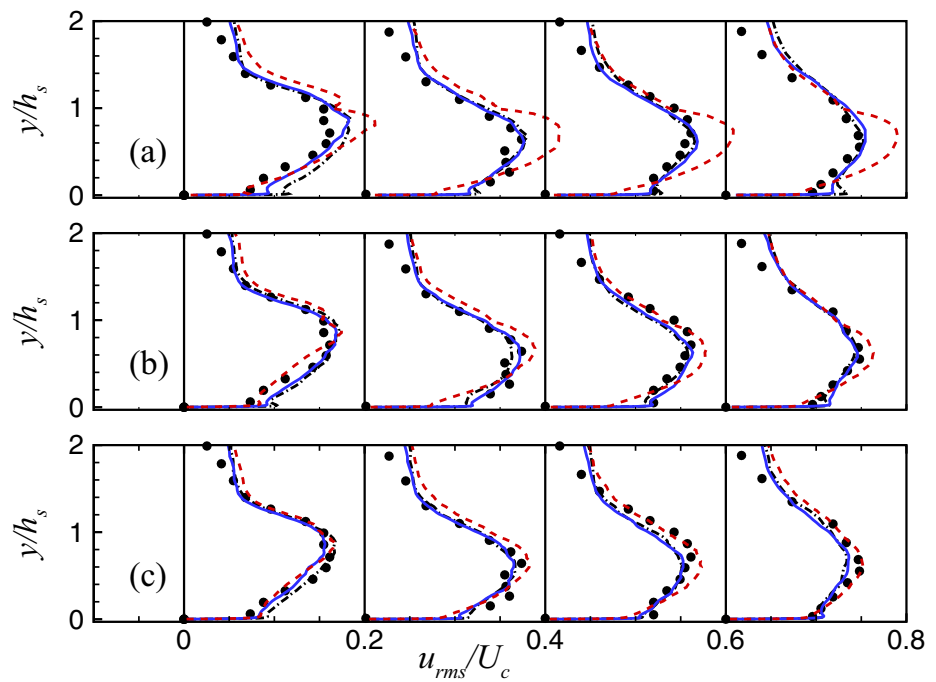

FIG. 13. Plots of $u_{\mathrm{rms}}$ velocity normalized by the centerline velocity at the inlet duct. The grid resolutions, locations, and legends are the same as in Fig. 12.

The global ILSA was also tested on the coarse resolution, using $C_{k}=0.0075$ and 0.013 , values that yield $s_{\tau}$ up to 0.03 in the first case and up to 0.05 in the second (the distribution of $s_{\tau}$ is of course nonuniform). The results are also in good agreement with the experimental data (not shown) and the case with the higher constant is only slightly less accurate.

Figure 15 shows the distributions of $L_{\text {est }}$ and $C_{k}$. The critical feature of the ILSA is the adaptation of $L_{\text {est }}$ to the local state of the flow. Near the edge of the step where small-scale structures emerge, $L_{\text {est }}$ decreases in size; then, after the separation, where the flow structures start growing in size, $L_{\text {est }}$ grows again. The dynamic variation of the model parameter is an additional property of the local
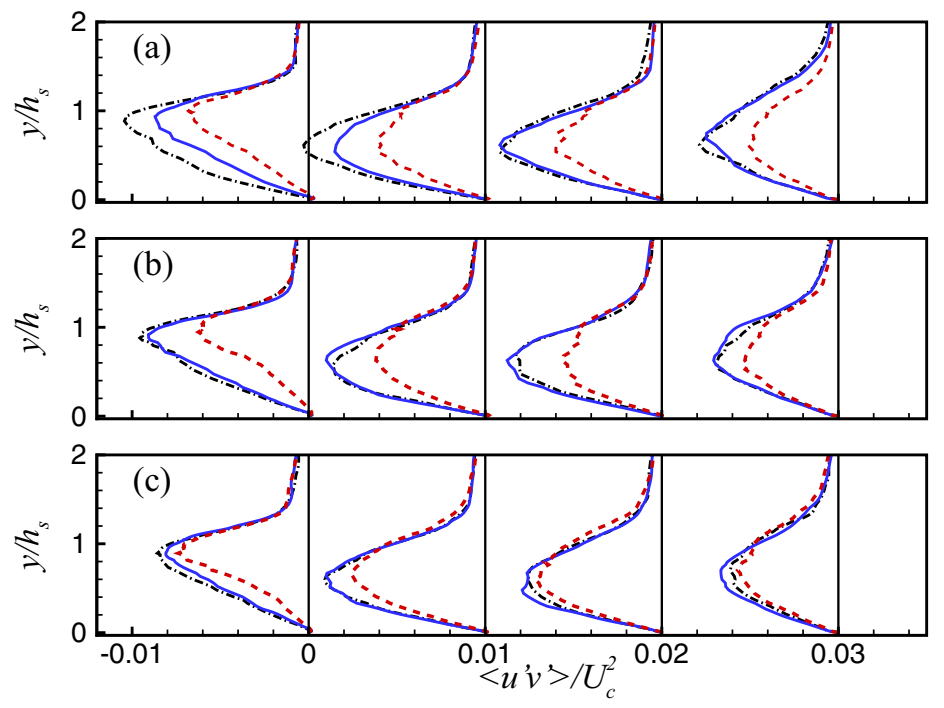

FIG. 14. Total Reynolds shear stress $\left\langle u^{\prime} v^{\prime}\right\rangle$ normalized by the centerline velocity at the inlet duct. The grid resolutions, locations, and legends are the same as in Fig. 12. 

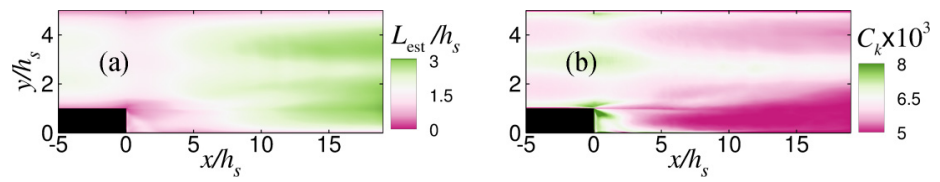

FIG. 15. Plots of the SFS parameters for the backward-facing step using the local ILSA on the coarse resolution $(256 \times 100 \times 64)$ for (a) $L_{\text {est }} / h_{s}$ and (b) $C_{k}$.

ILSA. After the separation region where $L_{\text {est }}$ is growing, $C_{k}$ decreases to maintain a constant value of the SFS activity.

The fact that the model length scale is based on the flow physics rather than on the grid is a major advantage in this problem, in which severe grid refinements are required to resolve appropriately the inviscid instability of the shear layer. Figure 16 compares the eddy viscosity obtained using the ILSA with that used in the dynamic model, which, because of its strong dependence on the grid size, is sharply reduced near the step. The ILSA, on the other hand, gives a smoother distribution of $v_{\mathrm{SFS}}$, since the length scale does not reflect the grid size but the turbulence properties only. The fact that the grid is refined does not, in this case, imply that, locally, finer scales are present. Because of the convective nature of this flow, the eddies present at a point in the separated shear layers are not generated locally, but advected from upstream. The decrease in eddy viscosity, not accompanied by an increase in the range of turbulent eddies present, is liable to result in errors of the type described by Vanella et al. [5] even when the mesh is not discontinuous. In more complex applications with sudden grid refinement, the discontinuity in the eddy viscosity would also lead to aliasing and commutation errors in the resolved scales [5].

\section{Effects of temporal averaging}

The local ILSA is formulated based on the integral scale, which relies on averaging the turbulence quantities [20]; in general, time averaging over an appropriate averaging period can be adopted in the simulations. However, in the problems described in this article, due to the existence of homogeneous directions, we used spatial averaging to evaluate the model parameters. The benefit of spatial averaging (especially for the channel, in which averaging is performed in both streamwise and spanwise directions) lies in the fact that several large eddies are included, resulting in fairly smooth TKE and total dissipation, thereby reducing numerical discretization error effects in addition to faster convergence in model parameters. In complex problems in which no homogeneous direction exists,
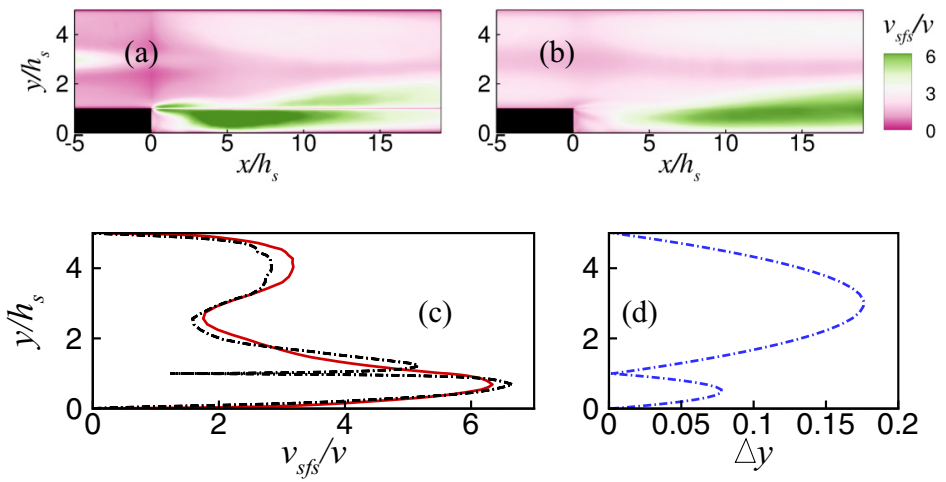

FIG. 16. Plots of the SFS eddy viscosity for the backward-facing step using coarse resolution $(256 \times 100 \times$ 64) for (a) the Lagrangian dynamic model [23], (b) the local ILSA with $s_{\tau}=0.022$, (c) the eddy viscosity across the backward-facing step at $x / h_{s}=10$ for the local ILSA with $s_{\tau}=0.022$ (red solid line) and the Lagrangian dynamic model (black dash-dotted line), and (d) the grid-size distribution across the backward-facing step. 
time is the only feasible averaging dimension as far as the evaluation of the SFS model is concerned. In this section we consider the effect of time averaging in detail. For a proper understanding it is important that one distinguishes clearly the standard long-time averaging needed to evaluate averaged properties during postprocessing from the weighted time averaging over much shorter time intervals (here denoted by $T_{\text {avg }}$ ) used to calculate the model parameters yielding the SFS flux in the simulation.

As part of the SFS model evaluation, we determine how sensitive the results are to $T_{\text {avg }}$. Using a large value of $T_{\text {avg }}$, the model might need a long transient before the simulations reach a statistically steady state; the flow may then adjust slowly to the model and the total computational time might be increased substantially. On the other hand, by using a small $T_{\text {avg }}$, error may appear in the long time-averaged mean and rms quantities due to the deviation of the SFS parameters from their integral nature. In this section we will examine the response of the model predictions when only temporal averaging is performed as part of the SFS model flux evaluation.

We consider a general formulation in which the average is performed over all previous times with an exponential weighting function

$$
\langle\phi\rangle(t)=\frac{1}{T_{\text {avg }}} \int_{-\infty}^{t} \phi\left(t^{\prime}\right) \exp \left[-\left(t-t^{\prime}\right) / T_{\text {avg }}\right] d t^{\prime},
$$

where $T_{\text {avg }}$ is the characteristic time scale of the averaging operation. This can be conveniently formulated, numerically, as

$$
\langle\phi\rangle^{n+1}=\epsilon \phi^{n+1}+(1-\epsilon)\langle\phi\rangle^{n}, \quad \epsilon=\frac{\Delta t}{\Delta t+T_{\mathrm{avg}}},
$$

where $\Delta t$ is the time step. For $T_{\text {avg }} \rightarrow 0$ this yields an instantaneous quantity (no averaging), while for $T_{\text {avg }} \rightarrow \infty$ the standard Reynolds average is recovered.

Equation (17) is used to evaluate all the averaged quantities in Eq. (5) and the model response to variations in $T_{\text {avg }}$ was studied in both channel flow and backward-facing step. For channel flow, we concentrated on $\operatorname{Re}_{\tau}=1000$ using $128 \times 129 \times 128$ grid points with $s_{\tau}=0.022$. The characteristic time $T_{\text {avg }}$ was based on the large-eddy turnover time $\delta / u_{\tau}$ and was varied from 0.0025 to 5 times $\delta / u_{\tau}$. Simulations were performed starting from a converged velocity field (the same in all cases) obtained from a previous simulation using the local ILSA with plane-averaging, initializing the eddy viscosity using the Smagorinsky model [3]; time histories at three points, located at $y^{+}=12,50$, and 800, were recorded. Data were collected every five time steps ( $\Delta t$ was about $\left.2.5 \times 10^{-4} \delta / u_{\tau}\right)$; to assess the convergence of the data in a consistent manner, cumulative averaging was carried out:

$$
\langle\phi\rangle_{\mathrm{cuml}}(t)=\frac{1}{t} \int_{0}^{t} \phi\left(t^{\prime}\right) d t^{\prime}
$$

Note that cumulative averaging yields a delayed convergence of the sample compared to averaging over a finite window, due to the effects of the initial transient. However, here we used this choice on purpose to assess how fast the initial transient is removed from the averaged quantities by changing $T_{\text {avg. }}$.

Results for the streamwise velocity, Reynolds shear stress, and eddy viscosity in the channel are shown in Fig. 17 at $y^{+}=12$ and 50; the behavior at $y^{+}=800$ was similar to that observed at $y^{+}=50$. Due to the initialization from a developed velocity field, first-order statistics were found to converge earlier (after about $20 \delta / u_{\tau}$ ) than second-order statistics and eddy viscosity. The local ILSA yields simultaneous convergence of turbulent fluctuations and SFS parameters, regardless of the choice of $T_{\text {avg }}$. For $T_{\text {avg }}=1.0 \delta / u_{\tau}-2.0 \delta / u_{\tau}$, after a transient of approximately $30 \delta / u_{\tau}$, both the eddy viscosity and Reynolds shear stress stabilize near the average value obtained using spatial averaging. The choice of $T_{\text {avg }}=5.0 \delta / u_{\tau}$ requires a longer time for convergence due to the memory of the initial transient, which is felt for a longer period. Overall, for values of $T_{\text {avg }}$ within the range $1.0 \delta / u_{\tau}-5.0 \delta / u_{\tau}$, the converged values are the same as the plane-averaged data. 

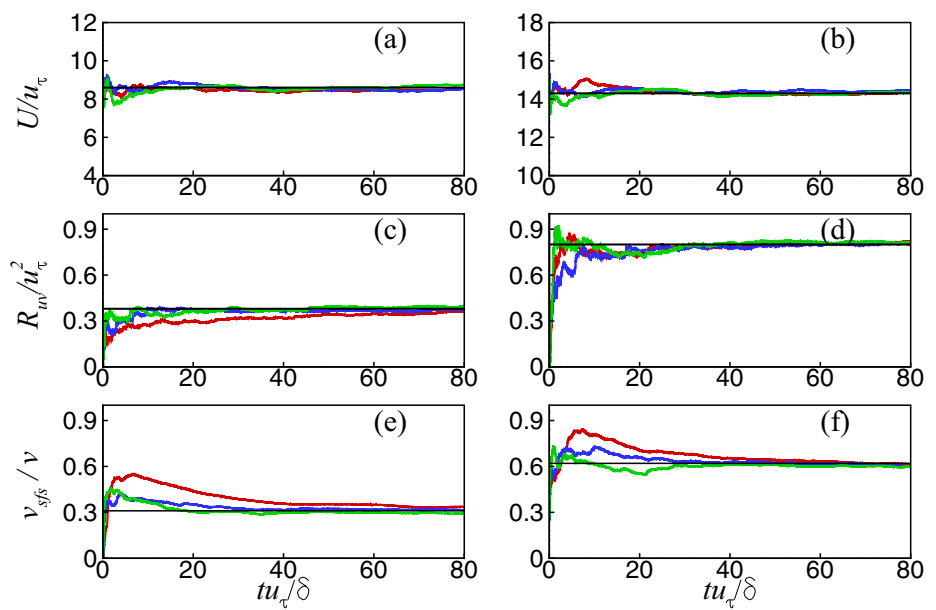

FIG. 17. History of (a) and (b) velocity $u$, (c) and (d) Reynolds shear stress $R_{u v}$, and (e) and (f) eddy viscosity for channel flow at (a), (c), and (e) $y^{+}=12$ and (b), (d), and (f) $y^{+}=50$ for $T_{\text {avg }}=5.0 \delta / u_{\tau}$ (red solid line), $T_{\text {avg }}=2.0 \delta / u_{\tau}$ (blue solid line), and $T_{\text {avg }}=1.0 \delta / u_{\tau}$ (green solid line). The straight horizontal line is the converged value from plane averaging.

The effect of $T_{\text {avg }}$ on the converged statistics is studied further in Figs. 18 and 19. Except for the case with the smallest characteristic time $T_{\text {avg }}=0.0025 \delta / u_{\tau}$, the eddy viscosity is insensitive to the choice of $T_{\text {avg }}$ (Fig. 18), as are the velocity statistics (Fig. 19). The smallest averaging time used reflects an almost instantaneous field, since it corresponds to averaging over nine or ten time steps only; when the averaging time becomes a small fraction of the integral time scale, the averaged quantities approach the instantaneous ones and thus the predicted fluctuating field (i.e., $\left.\phi^{\prime}=\phi-\langle\phi\rangle\right)$ approaches zero, which ultimately under-predicts the eddy viscosity. The fact that the eddy viscosity does not change as long as $T_{\text {avg }}$ is of the order of a large-eddy turnover time indicates that time averaging can be performed in flows without homogeneous directions. It should also be noted that the time required for the SFS quantities to converge is similar to that required by all the other statistics and the computational overhead is limited.

We performed the same study in the backward-facing step, which is more representative of flows with large-scale unsteadiness, since the recirculation bubble oscillates at a fairly low frequency [31]. We considered the case with $s_{\tau}=0.022$ using $384 \times 150 \times 96$ grid points. The shedding period of the separated shear layer $\tau_{s}$ was used as the integral time scale of the flow and is estimated as $\tau_{s}=X_{R} / U_{b}$, where $X_{R}$ is the separation length and $U_{b}$ is the bulk inflow velocity [32]. The time history of SFS parameters was recorded at six points, as $T_{\text {avg }}$ was varied from $\tau_{s}$ to $5 \tau_{s}$. Also

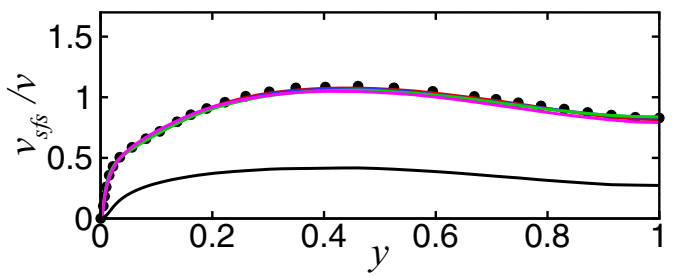

FIG. 18. Ratio of eddy viscosity to kinematic viscosity for channel flow at $\operatorname{Re}_{\tau}=1000$ with $s_{\tau}=0.022$ using $128 \times 129 \times 128$ grid points for $T_{\mathrm{avg}}=5.0 \delta / u_{\tau}$ (red solid line), $T_{\mathrm{avg}}=2.0 \delta / u_{\tau}$ (blue solid line), $T_{\mathrm{avg}}=$ $1.0 \delta / u_{\tau}$ (green solid line), $T_{\mathrm{avg}}=0.1 \delta / u_{\tau}$ (pink solid line), $T_{\mathrm{avg}}=0.0025 \delta / u_{\tau}$ (black solid line), and plane averaging (closed circles). 

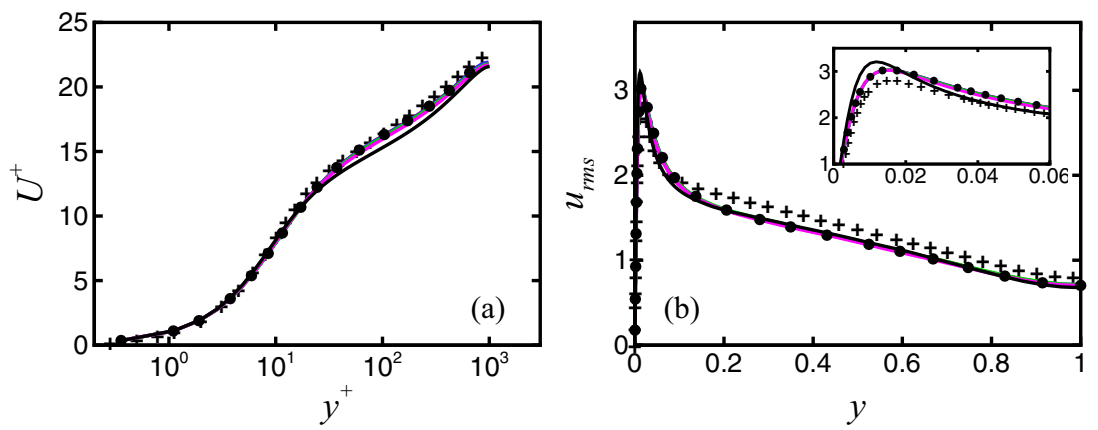

FIG. 19. (a) Mean streamwise velocity and (b) $u_{\mathrm{rms}}$ for channel flow at $\operatorname{Re}_{\tau}=1000$ with $s_{\tau}=0.022$ using $128 \times 129 \times 128$ grid points. Legends are the same as in Fig. 18 .

$T_{\text {avg }}=0.1 \tau_{s}$ was tested but, as in the channel, the use of nearly instantaneous data affected adversely the results and is not shown. Similar to the channel flow, simulations were initiated from a converged velocity field with the Smagorinsky eddy viscosity as the initial condition for $v_{\text {SFS }}$.

Figure 20 shows the location of the monitoring points and the history of the solution at two representative points [points (2) and (4)], one near the shear layer and the other one within the separation bubble. The second one can be expected to yield the slowest convergence, since it is not in an advection-dominated region, and the oscillations of the separation bubble are expected to affect the convergence of the statistics. According to Simpson [31], the size of the separation bubble oscillates (flapping motion) with a period that is about an order of magnitude larger than $\tau_{s}$.
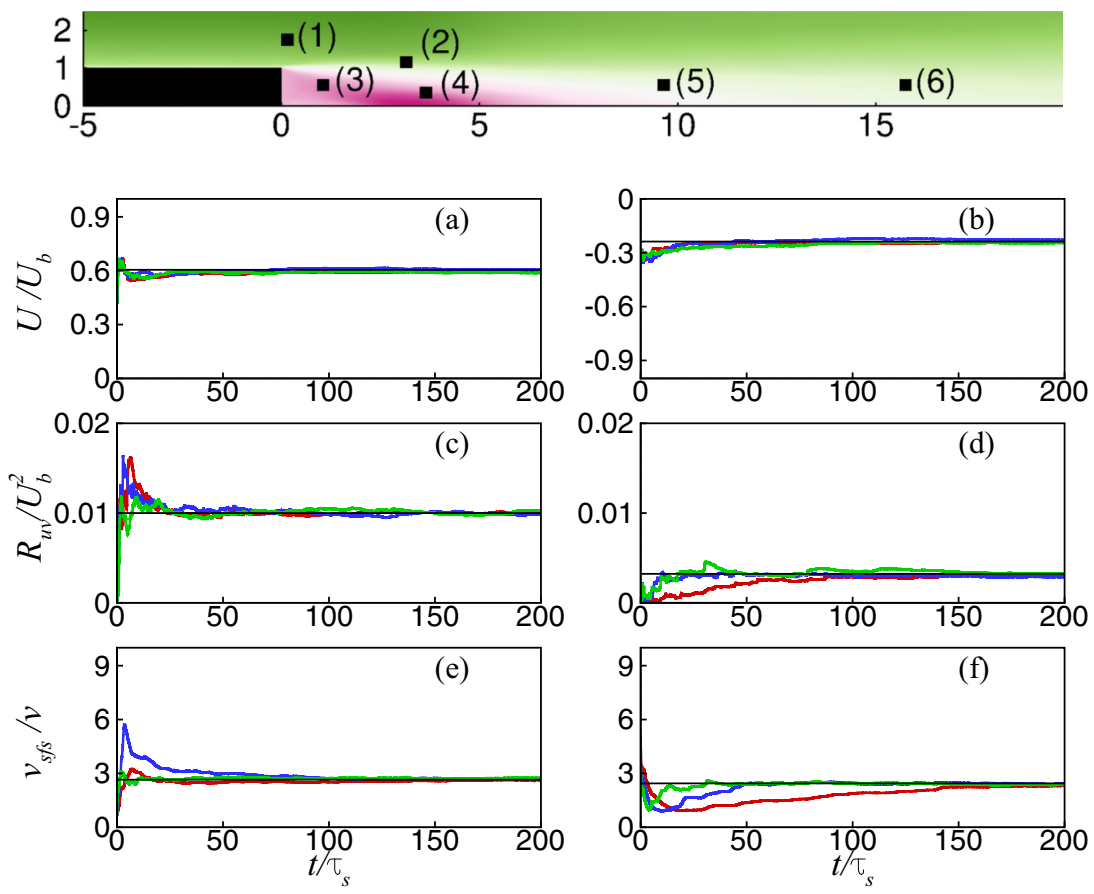

FIG. 20. History of (a) and (b) streamwise mean velocity, (c) and (d) Reynolds shear-stress, and (e) and (f) eddy viscosity for the backward-facing step with $s_{\tau}=0.022$ using $384 \times 150 \times 96$ grid points at (a), (c), and (e) point (2) and (b), (d), and (f) point (4) for $T_{\text {avg }}=5.0 \tau_{s}$ (red solid line), $T_{\text {avg }}=2.0 \tau_{s}$ (blue solid line), and $T_{\text {avg }}=1.0 \tau_{s}$ (green solid line). The straight horizontal line is the converged value from line averaging. 

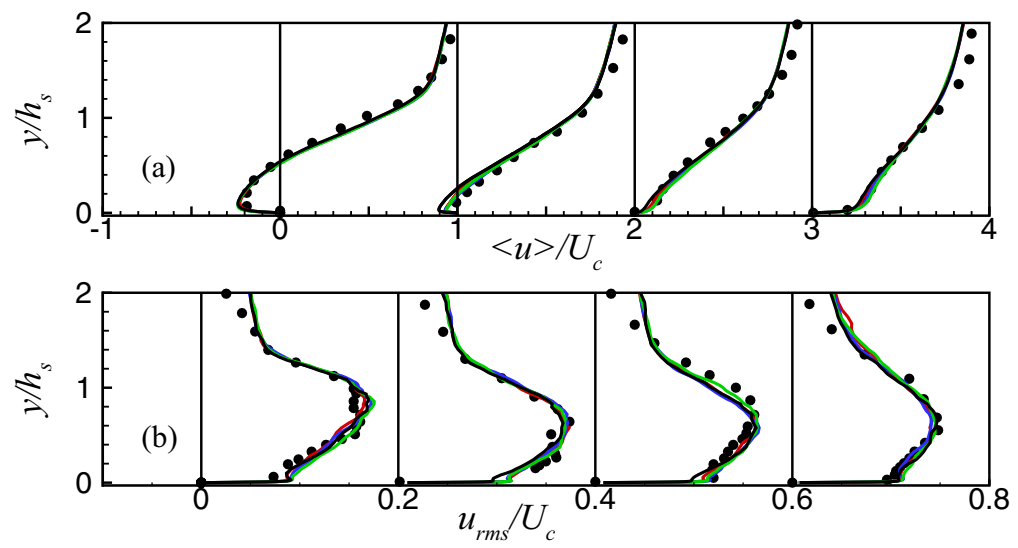

FIG. 21. (a) Mean streamwise and (b) $u_{\text {rms }}$ velocities normalized by the centerline velocity at the inlet duct for $T_{\text {avg }}=5.0 \tau_{s}$ (red solid line), $T_{\text {avg }}=2.0 \tau_{s}$ (blue solid line), $T_{\text {avg }}=1.0 \tau_{s}$ (green solid line), $T_{\text {avg }}=0.1 \tau_{s}$ (black solid line), and line averaging (closed circles). Locations are the same as in Fig. 12.

In Fig. 20 the cumulative-averaged statistics are plotted. At point (2) convergence of the results is insensitive to the choice of $T_{\text {avg }}$ and occurs at approximately $50 \tau_{s}$. It can be conjectured that evolution of the flow at this point is mainly dependent on the time scale of the inflow channel; the range of $T_{\text {avg }}=5.0 \tau_{s}-1.0 \tau_{s}$ is equivalent to about 0.75 to 0.15 of the large-eddy turnover time of the inflow duct. According to our channel flow analysis, this is the range within which fast convergence is reached and at the same time the averaged quantities can accurately yield the integral parameters.

As expected, point (4) in the recirculation bubble converges more slowly, especially for $T_{\text {avg }}=5 \tau_{s}$. However, for $T_{\mathrm{avg}}=1.0 \tau_{s}-2.0 \tau_{s}$, convergence is reached in approximately $t=50 \tau_{s}-20 \tau_{s}$. Within the recirculation bubble the time scale of the flow is mainly dictated by the oscillation period of the bubble; therefore the choice of $T_{\text {avg }}=1.0 \tau_{s}$ might be thought to be too low as an averaging window. However, this is equivalent to about 0.1 of the local integral time scale and, according to the channel flow analysis, this averaging time gives adequate results for the integral quantities. If we average the results over a finite time window (of the order of the integral time scale) rather than using cumulative averages, convergence is reached in about $10 \tau_{s}-20 \tau_{s}$ (not shown here).

For reference, for the production simulation of the backward-facing step starting from coarse simulation data, it takes about $15 \tau_{s}$ for the first-order statistics to converge, while the data shown for backward-facing step in the previous section were averaged over a time of about $75 \tau_{s}$. Since our analysis here indicates that little simulation time is required (over that required for turbulence statistics) for the convergence of the SFS quantities required in the model, we conclude that time averaging in the ILSA model would not increase the computational cost appreciably.

The sensitivity of the statistics to $T_{\text {avg }}$ is shown in Fig. 21. All the turbulence statistics collapse, except for $T_{\text {avg }}=0.1 \tau_{s}$, which presents a slight underprediction in the mean velocity near the reattachment point, due to the eddy viscosity, which is underpredicted by about $50 \%$ in that region. The choice of $T_{\mathrm{avg}}=0.1 \tau_{s}$ corresponds to 0.01 of the local time scale within the recirculation bubble, equivalent to a nearly instantaneous calculation of SFS parameters and causing a rise in the modeling error.

In summary, our analysis demonstrates that the local ILSA can be applied to complex problems using time as the only averaging dimension. With a time-averaging period in the order of the integral time scale of the flow, convergence of the SFS parameters is reached as fast as the flow field itself and at the same time sufficient sampling period is provided for accurate reconstruction of integral quantities that are used in the SFS model. 


\section{CONCLUSION}

We developed an eddy-viscosity model for LES, based on the one proposed by Piomelli et al. [7], which uses a model length scale based on an estimation of the integral scale of turbulence (the ILSA model). This model was used for simulations of plane channel flow and flow over a backward-facing step.

Two main modifications were carried out. First, a measure of subfilter activity was defined that is more robust to increases in the Reynolds number. This quantity $s_{\tau}$ measures the contribution of the subfilter scales to the second invariant of the Reynolds stress tensor. This quantity is related to the percentage of turbulent kinetic energy carried by the SFSs and the ratio between the two can be calculated for homogeneous isotropic turbulence at high Reynolds number. For low Reynolds numbers and in the presence of shear, the relationship is harder to quantify, but the ratio remains of order one.

A second improvement was carried out by making the model more responsive to the local dynamics of the flow. In the original ILSA model, the model coefficient $C_{k}$ was a constant, assigned in such a way to maintain a desired value of the SFS activity measure chosen. In the formulation called the local ILSA, $C_{k}$ is calculated dynamically, at each time step, to satisfy the desired SFS activity locally, using either local spatial averaging or time averaging. This modification has an additional advantage in that it improves the near-wall asymptotic behavior of the model.

Application of the local ILSA to channel flow indicated that $s_{\tau} \leqslant 0.03$ yields an accurate solution. Values of $s_{\tau}$ above this range cause the SFS shear stress to carry more than $50 \%$ of the Reynolds stress in the buffer layer, causing an increase in the modeling error. The model is at least as accurate, in channel flow, as the dynamic model [24] at a substantially reduced cost. On coarse or marginal meshes, the model is significantly more accurate, due to its better behavior near the wall.

The local ILSA was also applied to flow over a BFS. Results indicated that ILSA adapts itself to the local state of the flow, varying consistently with the size of the turbulent eddies. Furthermore, better control over local SFS activity resulted in higher accuracy of the local ILSA compared to the dynamic model on coarse grids. Finally, the distribution of the eddy viscosity demonstrated that, due to the minimal sensitivity of the ILSA to the grid topology, the local ILSA results in a smooth distribution of the eddy viscosity independent of the local grid size, whereas the grid-based nature of the dynamic model yields sharp variations of the eddy viscosity in the refined-grid region. The smooth but physically consistent model length scale produced by the ILSA model makes it a suitable choice for complex geometries with an unstructured grid in which sudden grid refinement might lead to an increase in the error if a grid-based filter width would be adopted [33,34].

In all presently used models, the user must provide a parameter that contains a certain degree of arbitrariness. In the Smagorinsky model, for instance, the choice is made of having $\Delta \propto h$ (the proportionality constant and the exact definition of $\Delta$ are also somewhat arbitrary, as discussed in Ref. [7]). The model constant is then calculated based on homogenous isotropic turbulence theory and modified to account for shear, solid walls, etc. In the dynamic model, the definition of the filter width $(\Delta \propto h)$ is still an arbitrary choice, as is the ratio between the test and grid filter. The model coefficient, however, is determined according to the dynamic procedure (which also has some degree of arbitrariness, caused by the type of averaging adopted). In the models based on the integral length-scale approximation the main choice the user must make is the expected cost of the calculation, measured by the SFS activity measure, in this case $s_{\tau}$. The smaller this number, the higher the cost of the simulation, as finer grids are required to resolve the smaller filter width associated with low values of the SFS activity measure. Once this choice has been made, the model coefficient is determined and the grid can be refined to achieve grid convergence. In a sense, ILSA models make the role of the computational cost of the calculation, and its relationship to the expected accuracy, explicit.

The local ILSA model has the potential of being a very adaptable, robust, and inexpensive tool for large-eddy simulations. Further studies should determine its accuracy in cases in which 
the perturbation of the turbulence from equilibrium is more significant (relaminarizing flows, for instance) and also in wall-modeled simulations.

\section{ACKNOWLEDGMENTS}

This research was supported by the Natural Sciences and Engineering Research Council under the Discovery Grant program. The authors thank the High Performance Computing Virtual Laboratory, Queen's University site, for the computational support. U.P. also acknowledges support from the Canada Research Chairs Program.

\section{APPENDIX: RELATIONSHIP BETWEEN $s_{\tau}$ AND $s_{k}$}

In this Appendix we use the theory of homogeneous isotropic turbulence to relate $s_{\tau}$ to $s_{k}$, whose meaning is easier to grasp intuitively. We begin by considering a model spectrum [20]

$$
\begin{gathered}
E(k)=\alpha \varepsilon^{2 / 3} k^{-5 / 3} f_{L}(k L) f_{\eta}(k \eta), \\
f_{L}(k L)=\left[\frac{k L}{\left[(k L)^{2}+6.78\right]^{1 / 2}}\right]^{11 / 3}, \\
f_{\eta}(k \eta)=\exp \left(-5.2\left\{\left[(k \eta)^{4}+0.40^{4}\right]^{1 / 4}-0.40\right\}\right) .
\end{gathered}
$$

The Kolmogorov constant is $\alpha=1.5, L=\left\langle\mathcal{K}_{\text {tot }}\right\rangle^{3 / 2} /\left\langle\varepsilon_{\text {tot }}\right\rangle$ is the dissipation length scale, $\eta=$ $L \operatorname{Re}_{L}^{-3 / 4}$ is the Kolmogorov scale, and $\operatorname{Re}_{L}=\left\langle\mathcal{K}_{\text {tot }}\right\rangle^{1 / 2} L / \nu$.

We use the box filter (in physical space) to filter the energy spectrum. The Fourier representation of the box filter is

$$
\widehat{G}(k)=\frac{\sin (k \Delta / 2)}{k \Delta / 2} .
$$

Then turbulent kinetic energy and dissipation are decomposed into resolved and subfilter parts

$$
\begin{gathered}
\left\langle\mathcal{K}_{\mathrm{SFS}}\right\rangle=\underbrace{\int_{0}^{+\infty} E(k) d k}_{\left\langle\mathcal{K}_{\text {tot }}\right\rangle}-\underbrace{\int_{\langle\text {res }}^{+\infty} \widehat{G}^{2}(k) E(k) d k}_{\left\langle\varepsilon_{\text {tot }}\right\rangle}, \\
\left\langle\varepsilon_{\mathrm{SFS}}\right\rangle=\underbrace{2 v \int_{0}^{+\infty} k^{2} E(k) d k}_{\left\langle\varepsilon_{\text {res }}\right\rangle}-\underbrace{2 v \int_{0}^{+\infty} k^{2} \widehat{G}^{2}(k) E(k) d k}
\end{gathered}
$$

and we perform the integrations varying the filter width $\Delta=C_{\Delta} L$ and $\operatorname{Re}_{L}$.

To calculate $s_{\tau}$, three terms must be computed, $\left\langle R_{i j}^{a} \tau_{i j}^{a}\right\rangle,\left\langle R_{i j}^{a} R_{i j}^{a}\right\rangle$, and $\left\langle\tau_{i j}^{a} \tau_{i j}^{a}\right\rangle$, which appear when the products in Eq. (8) are expanded. In a well-resolved LES, $\left|\tau_{i j}^{a}\right| \ll\left|R_{i j}^{a}\right|$; thus, $\left\langle R_{i j}^{a} \tau_{i j}^{a}\right\rangle$ and $\left\langle\tau_{i j}^{a} \tau_{i j}^{a}\right\rangle$ are expected to be significantly smaller than $\left\langle R_{i j}^{a} R_{i j}^{a}\right\rangle$ and can be neglected (an assumption that will be validated momentarily). An approximation of $s_{\tau}$ can then be written as

$$
s_{\tau}^{*}=\left[\frac{\left\langle\tau_{i j}^{a} \tau_{i j}^{a}\right\rangle}{\left\langle R_{m n}^{a} R_{m n}^{a}\right\rangle}\right]^{1 / 2} .
$$

By expanding $\left\langle R_{i j}^{a} R_{i j}^{a}\right\rangle$ we obtain

$$
\left\langle R_{i j}^{a} R_{i j}^{a}\right\rangle=\frac{2}{3}\left\langle\bar{u}_{i}^{\prime} \bar{u}_{i}^{\prime} \bar{u}_{j}^{\prime} \bar{u}_{j}^{\prime}\right\rangle
$$

The fourth moments can be expressed in terms of the second ones if the velocity fluctuations are assumed to behave as Gaussian functions; such behavior is supported by previous studies [35,36]. 

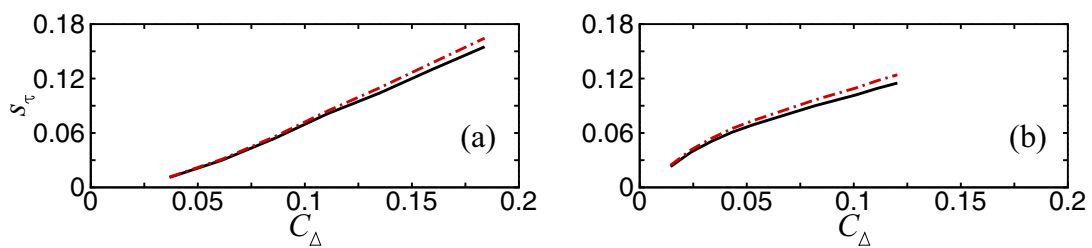

FIG. 22. Variation of $s_{\tau}$ (black solid line) and $s_{\tau}^{*}$ (red dash-dotted line) with $C_{\Delta}$ for filtered DNS of homogeneous isotropic turbulence at (a) $\operatorname{Re}_{L}=250$ and (b) $\operatorname{Re}_{L}=4490$.

Isserlis's theorem [37] for Gaussian random functions allows us to write

$$
\left\langle R_{i j}^{a} R_{i j}^{a}\right\rangle \simeq \frac{10}{9}\left\langle\bar{u}_{k}^{\prime} \bar{u}_{k}^{\prime}\right\rangle^{2}=\frac{40}{9}\left\langle\mathcal{K}_{\text {res }}\right\rangle^{2},
$$

which gives

$$
s_{\tau}^{*}=\left[\frac{\left\langle\tau_{i j}^{a} \tau_{i j}^{a}\right\rangle}{40 / 9\left\langle\mathcal{K}_{\mathrm{res}}\right\rangle^{2}}\right]^{1 / 2}
$$

We compared the values of $s_{\tau}$ and $s_{\tau}^{*}$ using our databases for homogeneous isotropic turbulence. The results are shown in Fig. 22 and indicate that the error due to neglecting the SFS contributions and assuming Gaussian behavior is less than $7 \%$.

To calculate the numerator of (A10), we use the eddy-viscosity hypothesis, consistent with the modeling approach used in this work:

$$
\left\langle\tau_{i j}^{a} \tau_{i j}^{a}\right\rangle=\left\langle 2 v_{\mathrm{SFS}}^{2}|\bar{S}|^{2}\right\rangle=2\left(C_{k} L\right)^{4}\left\langle|\bar{S}|^{4}\right\rangle
$$

While the ILSA model does not require that $\Delta$ is specified, here we need to determine it. Consequently, we write

$$
C_{k} L=C_{m} C_{\Delta} L
$$

and determine $C_{m}$, following Lilly [4] and Meyers and Sagaut [38], by equating the SFS dissipation modeled using the eddy viscosity with its exact value from (A6):

$$
\left\langle 2 v_{\mathrm{SFS}} \bar{S}_{i j} \bar{S}_{i j}\right\rangle=\left\langle\varepsilon_{\mathrm{SFS}}\right\rangle \Rightarrow C_{m}^{2}=\frac{\left\langle\varepsilon_{\mathrm{SFS}}\right\rangle}{\left(C_{\Delta} L\right)^{2}\left\langle|\bar{S}|^{3}\right\rangle} \simeq \frac{\left\langle\varepsilon_{\mathrm{SFS}}\right\rangle}{\left(C_{\Delta} L\right)^{2}\left\langle|\bar{S}|^{2}\right\rangle^{3 / 2}},
$$

where $\left\langle|\bar{S}|^{3}\right\rangle \simeq\left\langle|\bar{S}|^{2}\right\rangle^{3 / 2}$ was used $[4,38]$ and $\left\langle|\bar{S}|^{2}\right\rangle=\left\langle\varepsilon_{\text {res }}\right\rangle / \nu$.

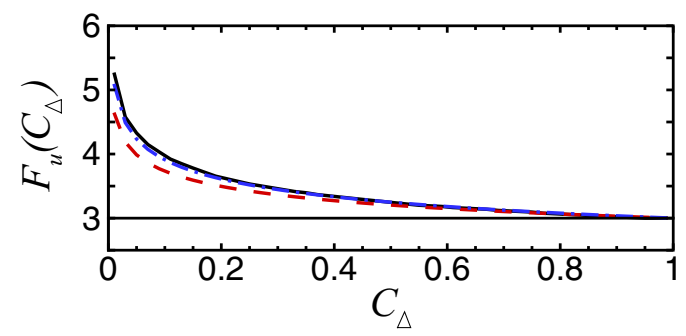

FIG. 23. Variation of flatness factor $F_{u}\left(C_{\Delta}\right)$ for the random $\beta$ model (black solid line) and empirical relations from DNS data [42] (red dashed line and blue dash-dotted line). 

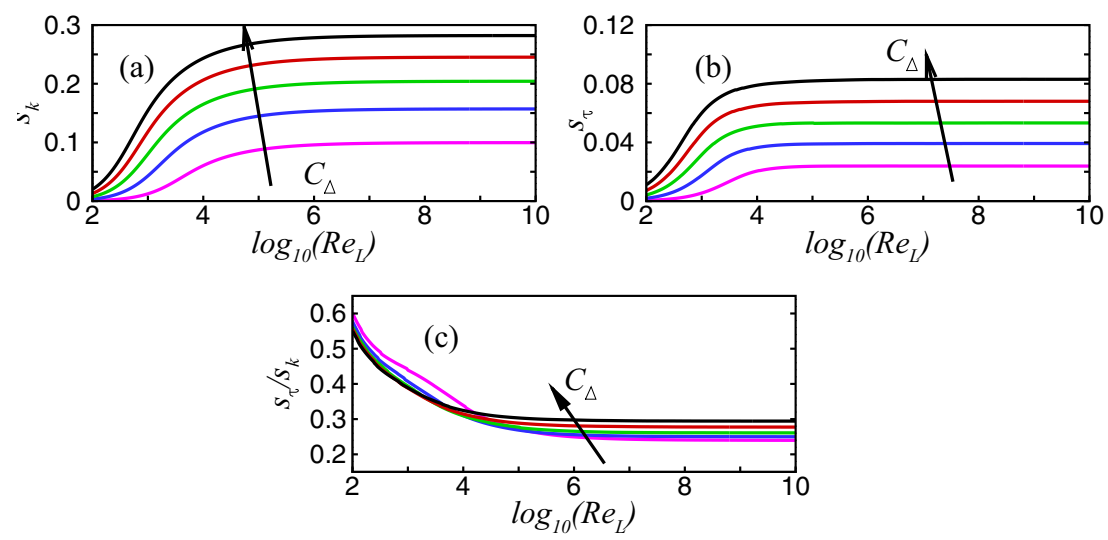

FIG. 24. Results of the model spectrum analysis for (a) $s_{k}$, (b) $s_{\tau}^{*}$, and (c) $s_{\tau}^{*} / s_{k}$. Each line corresponds to a constant $C_{\Delta}$ for $C_{\Delta}=0.02,0.04,0.06,0.08,0.10$.

The remaining parameter to be calculated in Eq. (A11) is $\left\langle|\bar{S}|^{4}\right\rangle$. Due to homogeneity and isotropy [39] we have

$$
\frac{\left\langle|\bar{S}|^{4}\right\rangle}{\left\langle|\bar{S}|^{2}\right\rangle^{2}}=\frac{7}{15} \frac{\left\langle\left(\partial \bar{u}_{1} / \partial x_{1}\right)^{4}\right\rangle}{\left\langle\left(\partial \bar{u}_{1} / \partial x_{1}\right)^{2}\right\rangle^{2}} .
$$

The Gaussian assumption for the velocity gradient is not valid due to intermittency effects $[35,36]$. Empirical relations for (A14) that include all the scales of turbulence [40] are not applicable to the filtered field. Instead, we take advantage of the box filter used and relate the filtered velocity gradient to the velocity structure function $\delta u_{1}(\Delta)$,

$$
\left(\frac{\partial \bar{u}_{1}}{\partial x_{1}}\right)=\overline{\left(\frac{\partial u_{1}}{\partial x_{1}}\right)}=\frac{1}{\Delta} \int_{x_{1}-\Delta / 2}^{x_{1}+\Delta / 2}\left(\frac{\partial u_{1}}{\partial x_{1}^{\prime}}\right) d x_{1}^{\prime}=\frac{\delta u_{1}(\Delta)}{\Delta} .
$$

Therefore, we have

$$
\frac{\left\langle\left(\partial \bar{u}_{1} / \partial x_{1}\right)^{4}\right\rangle}{\left\langle\left(\partial \bar{u}_{1} / \partial x_{1}\right)^{2}\right\rangle^{2}}=\frac{\left\langle\delta u_{1}^{4}(\Delta)\right\rangle}{\left\langle\delta u_{1}^{2}(\Delta)\right\rangle^{2}}=F_{u}(\Delta)
$$

To calculate $F_{u}(\Delta)$, we use the multifractal random $\beta$ model [41]. This method predicts the probability density function (PDF) of the velocity difference $\delta u\left(\Delta_{n}\right)$ at separation distances that are fractions of the integral length scale (i.e., $\Delta_{n}=L / a^{n}, a>1$ ). Then the fourth-order structure function $\left\langle\delta u^{4}\left(\Delta_{n}\right)\right\rangle$ was predicted through the integration of its PDF. The agreement between the $\beta$ model and the DNS data is very good, as shown in Fig. 23. The $F_{u}(\Delta)$ given by the model was substituted in Eqs. (A16) and (A14) to evaluate the ratio $\left\langle|\bar{S}|^{4}\right\rangle /\left\langle|\bar{S}|^{2}\right\rangle^{2}$. The $\left\langle|\bar{S}|^{2}\right\rangle$ can be calculated from the model spectrum and $\left\langle|\bar{S}|^{4}\right\rangle$ can be substituted into (A11) to obtain $s_{\tau}^{*}$. The $s_{k}$ can be calculated directly from (A5) and the model spectrum.

The results of this analysis are summarized in Fig. 24, which shows $s_{k}$ and $s_{\tau}^{*}$ for $10^{2} \leqslant \mathrm{Re}_{L} \leqslant$ $10^{10}$ and $0.02 \leqslant C_{\Delta} \leqslant 0.10$. For $\operatorname{Re}_{L}>10^{6}$ both $s_{\tau}^{*}$ and $s_{k}$ and their ratio become Reynolds number independent and are affected by $C_{\Delta}$ only. The ratio $s_{\tau} / s_{k}$ converges to $0.2-0.3$ for $\operatorname{Re}_{L} \geqslant 10^{6}$.

[1] A. Leonard, Energy cascade in large-eddy simulations of turbulent fluid flows, Adv. Geophys. 18, 237 (1975). 
[2] S. B. Pope, Ten questions concerning the large-eddy simulation of turbulent flows, New J. Phys. 6, 35 (2004).

[3] J. Smagorinsky, General circulation experiments with the primitive equations: I. The basic experiment, Mon. Weather Rev. 91, 99 (1963).

[4] D. K. Lilly, The representation of small scale turbulence in numerical simulation experiments, in Proceedings of the IBM Scientific Computing Symposium on Environmental Sciences, Yorktwon Heights, NY, edited by H.H. Goldstine, IBM Form No. 320-1951 (White Plains, NY, 1967), pp. 195-210.

[5] M. Vanella, U. Piomelli, and E. Balaras, Effect of grid discontinuities on large-eddy simulation statistics and flow fields, J. Turbul. 9(32) (2008).

[6] F. van der Bos and B. J. Geurts, Commutator errors in the filtering approach to large-eddy simulation, Phys. Fluids 17, 035108 (2005).

[7] U. Piomelli, A. Rouhi, and B. J. Geurts, A grid-independent length scale for large-eddy simulations, J. Fluid Mech. 766, 499 (2015).

[8] B. J. Geurts and J. Fröhlich, A framework for predicting accuracy limitations in large-eddy simulation, Phys. Fluids 14, L41 (2002).

[9] B. J. Geurts and F. van der Bos, Numerically induced high-pass dynamics in large-eddy simulation, Phys. Fluids 17, 125103 (2005).

[10] B. J. Geurts, Direct and Large-Eddy Simulation III (Springer, Berlin, 1999), pp. 1-12.

[11] B. J. Geurts and J. Meyers, Successive inverse polynomial interpolation to optimize Smagorinsky's model for large-eddy simulation of homogeneous turbulence, Phys. Fluids 18, 118102 (2006).

[12] C. Rosales and C. Meneveau, Linear forcing in numerical simulations of isotropic turbulence: Physical space implementations and convergence properties, Phys. Fluids 17, 095106 (2005).

[13] A. J. Chorin, Numerical solution of the Navier-Stokes equations, Math. Comput. 22, 745 (1968).

[14] J. Kim and P. Moin, Application of a fractional-step method to incompressible Navier-Stokes equations, J. Comput. Phys. 59, 308 (1985).

[15] R. A. Sweet, A generalized cyclic reduction algorithm, SIAM J. Numer. Anal. 11, 506 (1974).

[16] A. Keating, U. Piomelli, E. Balaras, and H.-J. Kaltenbach, A priori and a posteriori tests of inflow conditions for large-eddy simulation, Phys. Fluids 16, 4696 (2004).

[17] A. Keating, U. Piomelli, K. Bremhorst, and S. Nesić, Large-eddy simulation of heat transfer downstream of a backward-facing step, J. Turbul. 5, 1 (2004).

[18] A. Keating and U. Piomelli, A dynamic stochastic forcing method as a wall-layer model for large-eddy simulation, J. Turbul. 7(12) (2006).

[19] E. A. Fadlun, R. Verzicco, P. Orlandi, and J. Mohd-Yusof, Combined immersed-boundary finite-difference methods for three-dimensional complex flow simulations, J. Comput. Phys. 161, 35 (2000).

[20] S. B. Pope, Turbulent Flows (Cambridge University Press, Cambridge, 2006).

[21] R. D. Moser, J. Kim, and N. N. Mansour, Direct numerical simulation of turbulent channel flow up to $\operatorname{Re}_{\tau}=590$, Phys. Fluids 11, 943 (1999).

[22] C. Meneveau and T. S. Lund, On the Lagrangian nature of the turbulence energy cascade, Phys. Fluids 6 , 2820 (1994).

[23] C. Meneveau, T. S. Lund, and W. H. Cabot, A Lagrangian dynamic subgrid-scale model of turbulence, J. Fluid Mech. 319, 353 (1996).

[24] M. Germano, U. Piomelli, P. Moin, and W. H. Cabot, A dynamic subgrid-scale eddy viscosity model, Phys. Fluids 3, 1760 (1991).

[25] S. Hoyas and J. Jiménez, Scaling of the velocity fluctuations in turbulent channels up to $\operatorname{Re}_{\tau}=2003$, Phys. Fluids 18, 011702 (2006).

[26] J. Meyers and P. Sagaut, Is plane-channel flow a friendly case for the testing of large-eddy simulation subgrid-scale models? Phys. Fluids 19, 048105 (2007).

[27] A. Rasam, G. Brethouwer, P. Schlatter, Q. Li, and A. V. Johansson, Effects of modeling, resolution and anisotropy of subgrid-scales on large eddy simulations of channel flow, J. Turbul. 12(10) (2011).

[28] J. C. Vogel and J. K. Eaton, Combined heat transfer and fluid dynamic measurements downstream of a backward-facing step, J. Heat Transfer 107, 922 (1985). 
[29] I. Orlanski, A simple boundary condition for unbounded hyperbolic flows, J. Comput. Phys. 21, 251 (1976).

[30] D. You and P. Moin, A dynamic global-coefficient subgrid-scale model for large-eddy simulation of turbulent scalar transport in complex geometries, Phys. Fluids 21, 045109 (2009).

[31] R. L. Simpson, Turbulent boundary-layer separation, Annu. Rev. Fluid Mech. 21, 205 (1989).

[32] P. G. Spazzini, G. Iuso, M. Onorato, N. Zurlo, and G. M. Di Cicca, Unsteady behavior of back-facing step flow, Exp. Fluids 30, 551 (2001).

[33] B. J. Geurts and J. Fröhlich, Modern Simulation Strategies for Turbulent Flow (Edwards, Waterbury, 2001), pp. 309-330.

[34] B. J. Geurts, Interacting errors in large-eddy simulation: A review of recent developments, J. Turbul. 7(55) (2006).

[35] Z. S. She, E. Jackson, and S. A. Orszag, Scale-dependent intermittency and coherence in turbulence, J. Sci. Comput. 3, 407 (1988).

[36] K. Yoshimatsu, N. Okamoto, K. Schneider, Y. Kaneda, and M. Farge, Intermittency and scale-dependent statistics in fully developed turbulence, Phys. Rev. E 79, 026303 (2009).

[37] L. Isserlis, On a formula for the product-moment coefficient of any order of a normal frequency distribution in any number of variables, Biometrika 12, 134 (1918).

[38] J. Meyers and P. Sagaut, On the model coefficients for the standard and the variational multi-scale Smagorinsky model, J. Fluid Mech. 569, 287 (2006).

[39] J. Hierro and C. Dopazo, Fourth-order statistical moments of the velocity gradient tensor in homogeneous, isotropic turbulence, Phys. Fluids. 15, 3434 (2007).

[40] T. Ishihara, Y. Kaneda, M. Yokokawa, K. I. Itakura, and A. Uno, Small-scale statistics in high-resolution direct numerical simulation of turbulence: Reynolds number dependence of one-point velocity gradient statistics, J. Fluid Mech. 592, 335 (2007).

[41] R. Benzi, G. Paladin, G. Parisi, and A. Vulpiani, On the multifractal nature of fully developed turbulence and chaotic systems, J. Phys. A: Math. Gen. 17, 3521 (1984).

[42] Z. S. She, S. Chen, G. Doolen, R. H. Kraichnan, and S. A. Orszag, Reynolds Number Dependence of Isotropic Navier-Stokes Turbulence, Phys. Rev. Lett. 70, 3251 (1993). 